Noname manuscript No.

(will be inserted by the editor)

\title{
Electron-Ion Temperature Equilibration in Collisionless Shocks: the Supernova Remnant-Solar Wind Connection
}

\author{
Parviz Ghavamian • Steven J. Schwartz • \\ Jeremy Mitchell • Adam Masters • J. Martin \\ Laming
}

Received: date / Accepted: date

\begin{abstract}
Collisionless shocks are loosely defined as shocks where the transition between pre-and post-shock states happens on a length scale much shorter than the
\end{abstract}

Parviz Ghavamian

Department of Physics, Astronomy and Geosciences

Towson University

Towson,Maryland, USA, 21252

Tel.: $+1(410)-704-3137$

Fax: +1 (410)-704-3700

E-mail: pghavamian@towson.edu

Steven J. Schwartz

Department of Physics

Imperial College London

Tel.: +44(0)20-7594-7660

Fax: +44(0)20-7594-7772

E-mail: s.schwartz@imperial.ac.uk

Jeremy Mitchell

Department of Physics

Imperial College London

Tel.: +44(0)20-7594-7660

Fax: +44(0)20-7594-7772

E-mail: j.mitchell@imperial.ac.uk

Adam Masters

Institute of Space and Astronautical Science

Japan Aerospace Exploration Agency

Kanagawa 252-5210, Japan

Tel.: +81-50-3362-6079

E-mail: a.masters@stp.isas.jaxa.jp

J. Martin Laming

Space Science Division

Naval Research Laboratory

Code 7684, Washington, DC 20375

Tel.: +1 (202) 767-4415

E-mail: j.laming@nrl.navy.mil 
collisional mean free path. In the absence of collision to enforce thermal equilibrium post-shock, electrons and ions need not have the same temperatures. While the acceleration of electrons for injection into shock acceleration processes to produce cosmic rays has received considerable attention, the related problem of the shock heating of quasi-thermal electrons has been relatively neglected.

In this paper we review that state of our knowledge of electron heating in astrophysical shocks, mainly associated with supernova remnants (SNRs), shocks in the solar wind associated with the terrestrial and Saturnian bowshocks, and galaxy cluster shocks. The solar wind and SNR samples indicate that the ratio of electron temperature, $\left(T_{e}\right)$ to ion temperature $\left(T_{p}\right)$ declines with increasing shock speed or Alfvén Mach number. We discuss the extent to which such behavior can be understood via cosmic ray-generated waves in a shock precursor, which then subsequently damp by heating electrons. Finally, we speculate that a similar mechanism may be at work for both solar wind and SNR shocks.

Keywords Collisionless Shocks · Supernova Remnants · Solar Wind · Cosmic Ray Acceleration

\section{Introduction}

Shock waves have been observed in a wide range of environments outside the Earth, from the solar wind to the hot gas in galaxy clusters. However, the mechanism whereby the gas in these environments is shocked has been poorly understood. While shock transitions in the Earth's atmosphere are mediated by molecular viscosity (and hence direct particle collisions), those in interstellar space and the solar wind are too dilute to form in this way. In non-relativistic shocks, the role of collisions is effectively played by collective interactions of the plasma with the magnetic field. This results in a multiscale shock transition having sub-structure at ion kinetic length scales (Larmor radius or inertial length) and potentially electron kinetic scales (inertial lengths or whistler mode) (e.g., Schwartz et al. (this volume), Treumann 2009). Such plasmas are termed collisionless. The magnetic fields threading through the charged particle plasmas in space endow the plasmas with elastic properties, much like a fluid. The kinetic energy of the inflowing gas is dissipated within this fluid via collective interactions between the particles and magnetic field, transferring energy from the magnetic field to the particles. The collective processes are the result of the DC electromagnetic fields present in the shock transition layer, kinematic phase mixing, and also plasma instabilities; the last give rise to a rich range of plasma waves and turbulent interactions.

It has long been known that these processes may heat the electrons beyond the mass-proportional value predicted by the Rankine-Hugoniot jump conditions - ample evidence is found in spacecraft studies of solar wind shocks (Schwartz et al. 1988) and multi wavelength spectroscopy of supernova remnants (Ghavamian et al. 2001, 2002, 2003, 2007; Laming et al. 1996; Rakowski et al. 2008) and galaxy cluster gas (Markevitch et al. 2005; Markevitch \& Vikhlinin 2007; Russell et al. 2012) understanding how this process depends on such shock parameters as shock speed, preshock magnetic field orientation and plasma beta has been slow.

In collisionless plasmas, the downstream state of the plasma cannot be uniquely determined from the upstream parameters because the Rankine-Hugoniot jump conditions only predict the total pressure downstream, not the individual contributions from 
the electrons and ions: $P=n_{i} k T_{i}+n_{e} k T_{e}$. At the limit of a strong shock, $n_{e}$ and $n_{i}$ are each 4 times their preshock values, so the relative values of $T_{e}$ and $T_{i}$ immediately behind the shock are wholly dependent upon the nature of the collisionless heating processes occurring at the shock transition. Although an MHD description can be used to describe the behavior of the gas far upstream and far downstream of the shock, a more detailed kinetic approach is required for understanding how the dissipation at the shock front transfers energy from plasma waves and turbulence to the electrons and ions.

Non-relativistic collisionless shocks can be broadly sorted into three categories: slow, intermediate and fast. The three types are defined according to the angle between the shock velocity and upstream magnetic field, as well as the relative value of the shock speed compared to the upstream sound speed $\left(c_{s} \equiv \sqrt{\gamma P / \rho}\right)$ and Alfvén speed $\left(v_{A} \equiv B / \sqrt{4 \pi \rho_{i}}\right)$. Most astrophysical shocks are quasi-perpendicular (i.e., they propagate at a nearly right angle to the preshock magnetic field), allowing only for fast-mode propagation. In that case, the relevant quantity is the magnetosonic Mach number, $M_{m s}\left(\equiv v_{s h} / \sqrt{v_{A}^{2}+c_{s}^{2}}\right)$. Collisionless shocks have also been classified according to whether the flow speed exceeds the sound speed in the downstream plasma (Kennel 1985). Above the critical Mach number where the flow is subsonic, the dissipation of flow energy into thermal energy can no longer be maintained by electrical resistivity, and plasma wave turbulence (the cause of which are instabilities generated when the electron and ion distribution functions are distorted at the shock transition) is required (Kennel 1985). Shocks above the critical Mach number are termed supercritical, while those below are termed subcritical. Note that even for subcritical shocks, observations suggests that kinetic processes other than resistivity and turbulence contribute to the shock dissipation (Greenstadt and Mellott 1987).There are also other, higher critical Mach numbers related to the formation of subshocks (Kennel et al. 1985) and non-steady cyclic shock reformation beyond the whistler critical Mach number (Krasnoselskikh et al. 2002). The critical Mach number for quasi-perpendicular shocks is estimated to be rather low $\sim 2.8$ (Edmiston and Kennel 1984). The Mach numbers of most SNR shocks are expected to be well in excess of this value, meaning that they are both fast-mode and supercritical.

As one approaches the high Mach numbers expected for astrophysical shocks $\left(M_{A} \sim\right.$ 20-100 for an ambient magnetic field of $3 \mu G$ ), a greater and greater fraction of the incoming ions become reflected back upstream from the shock front, corresponding to an increasingly turbulent and and disordered shock transition. Physical parameters such as $B, T$ and $n$ no longer jump in an ordered manner (i.e., the transitions are no longer laminar). In addition to the reflected particles, the hot ions from downstream become hot enough to escape upstream, further enhancing the population of ions in front of the shock. These ions, which form a precursor, are now believed to play an essential role in the dissipation of high Mach number collisionless shocks. Aside from providing the seed population for the acceleration of cosmic rays, the precursor ions are likely generate a variety of plasma waves capable of selectively heating the electrons over the ions, thereby providing an important mechanism for raising $T_{e} / T_{p}$ above the mass-proportional value of $m_{e} / m_{p}$. 


\section{Formalism: Equilibration Timescales}

Taken at face value, the Rankine-Hugoniot jump conditions predict that electrons and ions will be heated in proportion to their masses:

$$
k T_{e, i}=\frac{3}{16} m_{e, i} V_{s h}^{2}
$$

In collisionless shocks, there are three relevant timescales to consider: the time required for Coulomb collisions to isotropize a distribution of electrons, $t_{e e}$, the time required for Coulomb collisions to isotropize distribution of ions, $t_{i i}$, and finally the time required for the electrons and ions to equilibrate to a common temperature, $t_{e i}$ (Spitzer 1962). After a time scale $t_{e e}$ or $t_{i i}$, the particles in question attain a Maxwellian velocity distribution. The self-collision time, $t_{c, e e}$ for electrons of density $n_{e}$ and temperature $T_{e}$ is (Spitzer 1962):

$$
t_{c, e e}=\frac{0.266 T_{e}^{3 / 2}}{n_{e} \ln \Lambda_{e}} \sec \approx \frac{0.0116 V_{s}(1000)^{3}}{n_{e} \ln \Lambda_{e}} y r
$$

where $\ln \Lambda$ is the Coulomb logarithm, Equation 1 has been used to write the relaxation time in terms of the shock speed and $V_{S}(1000)$ is the shock speed in units of 1000 $\mathrm{km} \mathrm{s}^{-1}$. For a young SNR having $V_{s} \sim 1000 \mathrm{~km} \mathrm{~s}^{-1}$, postshock density $\mathrm{n} \sim 1 \mathrm{~cm}^{-3}$ and $\ln \Lambda \sim 30$ the time required to establish a Maxwellian distribution at the electron temperature given by equation 1 is $t_{c, e e} \sim 0.004$ yrs. The electrons are isotropized by self-collisions first, then the protons, and finally over a longer timescale the electrons and protons equilibrate to a common temperature. For this reason, Coulomb collisions alone are unable to establish equilibration electron and proton distributions at the shock front. This equilibration is described by the relation

$$
\frac{d T_{e}}{d t}=\frac{T_{p}-T_{e}}{t_{c, e p}}
$$

where

$$
t_{c, e p}=\sqrt{\frac{m_{p}}{m_{e}}} t_{c, p p}=\frac{m_{p}}{m_{e}} t_{c, e e}
$$

The temperatures $T_{e}$ and $T_{i}$ equilibrate to a common density-weighted average temperature $T_{a v}$, given by $T_{a v}=\frac{3}{16} \mu m_{p} V_{s}^{2}$, where $\mu$ is the mean mass per particle $(=$ $(1.4 / 2.3)=0.6$ for cosmic abundances $)$. For mass-proportional heating, the timescale given by Equation 4 is $\sim 2000$ yrs for $V_{s} \geq 1000 \mathrm{~km} \mathrm{~s}^{-1}$, of similar order but longer than the proton-proton isotropization timescale, $t_{c, p p}$. These are longer than the age of the SNR, substantially so at higher $V_{s}$ indicating that for minimal heating (i.e., $\left.T_{e} / T_{p}=\frac{m_{e}}{m_{p}} \sim 1 / 1836\right)$ the electrons and ions will not equilibrate to $T_{a v}$ during the lifetime of the SNR (Itoh 1978; Draine \& McKee 1993).

The arguments above indicate that Coulomb collisions are ineffective at both isotropizing the heavy ion particle distributions and equilibrating the electron and ion temperatures at the transtions of collisionless shocks. The emission spectra of nonradiative SNRs (dominated mostly by X-ray and ultraviolet emission) should therefore be sensitive probes of the collisionless heating processes at the shock transition. We consider the observational constraints of these processes below. 


\section{Observational Constraints From SNRs}

The most useful shocks for studying collisionless equilibration processes are those exhibiting detectable emission from the immediate postshock gas. To be diagnostically useful, the emission should arise from the region close to the shock front, where temperature disequilibrium between electrons, protons and heavy ions is substantial enough to affect both the relatives fluxes and relative velocity widths of emission lines. The shape and extent of the spatial profile for the different emission species behind the shock is also a useful diagnostic, especially for the UV resonance lines of He II $\lambda 1640$, C IV $\lambda \lambda 1548,1550$, N V $\lambda \lambda 1238,1243$, and O VI $\lambda 1032,1038$. For a given shock speed, the distance behind the shock where the emission peaks depends strongly on the initial electron temperature, and electron temperature immediately behind the shock.

The ubiquity of non-radiative SNRs, as well as their relatively simple geometry and very high shock speeds, makes these objects the most important laboratories for investigating the efficiency and nature of electron-ion and ion-ion equilibration. Other non-radiative shocks available for study are those occurring in stellar wind bubbles (for example, Wolf-Rayet bubbles) (Gosset et al. 2011) and in galaxy cluster shocks (e.g., the 'Bullet Cluster' (Markevitch et al. 2005) and Abell 2146 (Russell et al. 2012)). However, these shocks are far less frequently observed than those in SNRs, leaving the latter as the most important objects providing both a broad range of shock speeds and corresponding diagnostic information (such as proper motions and spatially resolved structure). Shocks in star forming regions, such as $\mathrm{HH}$ objects and their associated bow shocks, tend to be clumpy and complicated, and are usually located in dense environments $\left(n \sim 100-1000 \mathrm{~cm}^{-3}\right)$ with low enough shock speeds $\left(V_{s} \sim 100-200 \mathrm{~km} \mathrm{~s}^{-1}\right)$ to be radiative. In those cases, the optical and UV emission (where the most valuable shock diagnostic line emission arises) is dominated by emission from the cooling and recombination zones far downstream from the shock. In those regions the cooling of the gas below $100,000 \mathrm{~K}$ and the accompanying compression of the gas result in a collisional plasma with $T_{e}=T_{p}=T_{i}$. This erases any 'memory' of the initial electron-ion equilibration. Furthermore, a significant fraction of the Ly $\alpha$ continuum produced in the recomination zone is expected to pass upstream and ionize the preshock gas. In strong shocks $\left(V_{s}>80 \mathrm{~km} \mathrm{~s}^{-1}\right)$, this results in complete ionization of hydrogen (Shull \& McKee 1979; Cox \& Raymond 1985; Dopita et al. 1993), thus precluding the use of collisionally excited Balmer line emission from neutral $\mathrm{H}$ (described in the next section) as a temperature equilibration diagnostic.

\subsection{Optical Spectroscopy of Balmer-Dominated Shocks: The $T_{e} / T_{p} \propto \mathrm{V}_{s h}^{-2}$ Relation}

In the late 1970 s it was discovered that very fast $\left(\sim 2000 \mathrm{~km} \mathrm{~s}^{-1}\right)$ shocks in young SNRs could generate detectable optical emission very close to the shock transition (Chevalier \& Raymond 1978; Chevalier, Kirshner \& Raymond 1980), providing a valuable diagnostic of physical conditions at the shock before Coulomb collisions or cooling could alter them. This emission is produced by collisional excitation of $\mathrm{H} \mathrm{I}$ as it flows into the shock front. The cold neutral component does not interact directly with the plasma waves and turbulence at the shock, while the ionized component is strongly heated and compressed by a factor of four (when the shock is strong). Some of the cold H is destroyed by collisional ionization; however, the rest of the cold $\mathrm{H}$ undergoes charge exchange with hot ions behind the shock, generating a separate population of hot $\mathrm{H}$. 


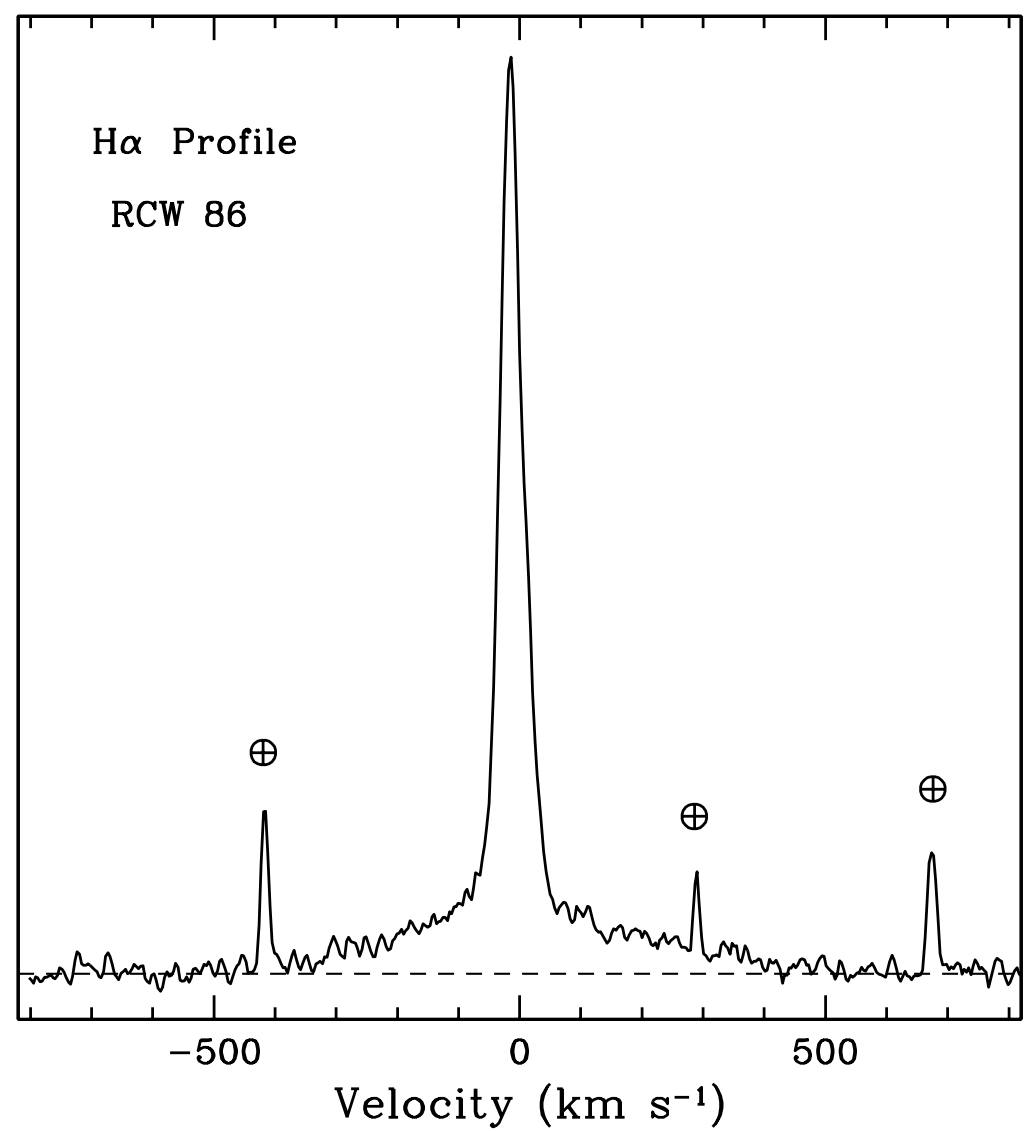

Fig. 1 The $\mathrm{H} \alpha$ line profile of a Balmer-dominated shock in RCW 86, acquired with a high dispersion (resolution $\left.\sim 10 \mathrm{~km} \mathrm{~s}^{-1}\right)$ spectrograph (Sollerman et al. 2003). The broad $(\sim 500$ $\left.\mathrm{km} \mathrm{s}^{-1}\right)$ and narrow $\left(\sim 30 \mathrm{~km} \mathrm{~s}^{-1}\right) \mathrm{H} \alpha$ lines are both resolved. Night sky lines (marked by the circles) have been left in to demonstrate their noticeably narrower width compared to the $\mathrm{H} \alpha$ lines

Approximately 1 in every 5 collisions results in collisional excitation to the $\mathrm{n}=3$ level of $\mathrm{H}$, producing $\mathrm{H} \alpha$ and Ly $\beta$ emission. The $\mathrm{H} \alpha$ line from the cold neutrals is narrow and reflects the preshock temperature $(\leq 30,000 \mathrm{~K})$, while that from the hot neutrals is broad (typically $\geq 500 \mathrm{~km} \mathrm{~s}^{-1}$ ), and reflects the postchock temperature (and to a large extent, the velocity distribution) of the protons (Chevalier \& Raymond 1978; Chevalier, Kirshner \& Raymond 1980; Smith et al. 1991; Ghavamian et al. 2001) (Figure 11). In Balmer-dominated shocks, the broad to narrow $\mathrm{H} \alpha$ flux ratio is proportional to the ratio of the charge exchange rate to the ionization rate, with the latter being highly sensitive to the electron and proton temperatures. This makes the broad to narrow ratio, $I_{b} / I_{n}$, very sensitive to $T_{e} / T_{p}$. There is only weak dependence of $I_{b} / I_{n}$ on the preshock H I fraction and preshock temperature, mainly due to differences in the amount of Ly $\beta$ converted into $\mathrm{H} \alpha$ in the narrow component (Ghavamian et al. 2001; van Adelsberg et al. 2008). 
The first systematic attempt to use Balmer-dominated SNRs to infer $T_{e} / T_{p}$ for collisionless shocks was attempted by Ghavamian et al. (2001). Using the broad H $\alpha$ line widths measured from a sample of Balmer-dominated shocks, they constrained the range of plausible shock speeds between the limits of minimal equilibration and full equilibration. They then predicted the broad-to-narrow ratios for a grid of shock models over this range of $V_{s}$ and $T_{e} / T_{p}$, allowing $T_{e} / T_{p}$ to be constrained. The range of broad component $\mathrm{H} \alpha$ widths observed in SNRs ranges from $\sim 250 \mathrm{~km} \mathrm{~s}^{-1}$ for the slowest Balmer-dominated shocks (Cgynus Loop), to $\sim 500 \mathrm{~km} \mathrm{~s}^{-1}$ for intermediatevelocity shocks (RCW 86) and finally $\sim 2600 \mathrm{~km} \mathrm{~s}^{-1}$ for the fastest shocks (SNR 0509-67.5). This corresponds to a well-sampled range of shock speeds: nearly a factor of 10. The primary uncertainty in measurement of the broad component width at low shock speeds $\left(\lesssim 200 \mathrm{~km} \mathrm{~s}^{-1}\right.$, as seen in the Northeastern Cygnus Loop; Hester et al. 1994) is disentangling the broad and narrow components when they are of comparable width. At high shock speeds $\left(\gtrsim 2000 \mathrm{~km} \mathrm{~s}^{-1}\right)$ the main difficulty is the baseline uncertainty of the surrounding continuum: if the peak of the broad line is low and the width very large, errors in ascertaining where the broad line merges into the background can lead to underestimates of the broad component width. The range of $I_{b} / I_{n}$ for this sample of Balmer-dominated shocks lies between 0.4 and 1.2 (Kirshner, Winkler \& Chevalier 1987; Smith et al. 1991; Ghavamian et al. 2001; 2003, Rakowski, Ghavamian \& Laming 2009). However, it does not not vary with broad component width in a monotonic fashion.

Recently there has been substantial improvement in the modeling of Balmer-dominated shocks. Earlier calculations of the broad $\mathrm{H} \alpha$ line profile assumed that it formed from a single charge exchange (Chevalier, Kirshner \& Raymond 1980; Smith et al. 1991; Ghavamian et al. 2001), and it treated the hot and cold neutrals as two separate, distinct populations, with a given neutral belonging to either one or the other. However, in reality an interaction 'tree' is required to track the number of photons emitted by each neutral over multiple excitations and charge exchanges. These effects were first incorporated in the Balmer-dominated shock models of Heng \& McCray (2007), who also found that charge exchange results in a third population of neutrals having velocity widths intermediate between the hot and cold neutrals. Further improvements in modeling of Balmer-dominated shocks were included by van Adelsberg et al. (2008), who included the momentum transferred by charge exchange between the hot neutrals and protons. This allowed the bulk velocity of the postshock neutrals to be calculated separately from those of the protons. Inclusion of the momentum transfer showed that for shock speeds $\lesssim 1000 \mathrm{~km} \mathrm{~s}^{-1}$, charge exchange effectively couples the fast neutral and thermal proton distributions, while for high shock speeds $\left(\gtrsim 5000 \mathrm{~km} \mathrm{~s}^{-1}\right)$, it does so far less effectively. This results in a fast neutral distribution that is skewed relative to the protons in velocity space and an average velocity that is much higher for the fast neutrals than the protons (van Adelsberg et al. 2008). Together, the inclusion of all these effects has enhanced the ability of the models to match the observed broadto-narrow ratios (and hence predict $T_{e} / T_{p}$ ). In particular, the newer models can now match the low broad-to-narrow ratio $\left(I_{b} / I_{n} \approx 0.67\right)$ observed in Knot g of Tycho's SNR, yielding $T_{e} / T_{p} \approx 0.05, V_{s} \approx 1600 \mathrm{~km} \mathrm{~s}^{-1}$. However, even after all the additional physics is included, the Balmer-dominated shock models are still unable to reach the low broadto-narrow ratios measured along the rims of DEM L71 $\left(I_{b} / I_{n} \approx 0.2-0.7\right.$; Ghavamian et al. 2003; Rakowski, Ghavamian \& Laming 2009). The electron-ion equilibration in DEM L 71 was instead determined via comparison of broad $\mathrm{H} \alpha$ line FWHM with postshock electron temperatures measured from Chandra observations (Rakowski, Ghavamian \& 
Hughes 2003). The most promising explanation advanced for the $I_{b} / I_{n}$ discrepancy has been added narrow component flux from the shock precursor (Raymond et al. 2011; Morlino et al. 2012), hitherto not included in the earlier shock models (Ghavamian et al. 2003, Rakowski, Ghavamian \& Laming 2009). These developments are described in more detail in the next section.

The plot of $T_{e} / T_{p}$ versus $V_{s}$ for the available sample of Balmer-dominated shocks shows a declining trend of equilibration with shock speed (Ghavamian et al. 2007; Heng et al. 2007; van Adelsberg et al. 2008). The trend is described by Ghavamian et al. (2007) as full equilibration for shock speeds up to and including $400 \mathrm{~km} \mathrm{~s}^{-1}$, and a declining equilibration proportional to the inverse square of the shock speed above 400 $\mathrm{km} \mathrm{s}^{-1}$. This description can be characterized in the following way:

$$
\frac{T_{e}}{T_{p}}=\left\{\begin{array}{cl}
1 & \text { if } \mathrm{V}_{\mathrm{s}}<400 \mathrm{~km} \mathrm{~s}^{-1} \\
\frac{m_{e}}{m_{p}}+\left(1-\frac{m_{e}}{m_{p}}\right)\left(\frac{V_{s}}{400}\right)^{-2} & \text { if } \mathrm{V}_{\mathrm{s}} \geq 400 \mathrm{~km} \mathrm{~s}^{-1}
\end{array}\right\}
$$

where the functional form of the the $T_{e} / T_{p}$ relation is designed to asymptotically transition to $T_{e} / T_{p}=m_{e} / m_{p}$ at very high shock velocities.

The most up to date plot of $T_{e} / T_{p}$ versus shock speed, reproduced from van Adelsberg et al. (2008), is shown in Figure 2 (Note that the shock models used in producing these plots do not include contribution from the shock precursor). Although the inverse correlation between $T_{e} / T_{p}$ and $V_{s}$ was largely confirmed by van Adelsberg et al (2008), there may be some evidence of departure from the $T_{e} / T_{p} \propto V_{s}^{-2}$ relation at shock speeds exceeding $2000 \mathrm{~km} \mathrm{~s}^{-1}$. van Adelsberg et al. find that when all three measured broad component widths and broad-to-narrow ratios from SN 1006 are included in the plot $\left(V_{s} \sim 2200-2500 \mathrm{~km} \mathrm{~s}^{-1}\right)$, a slight upturn in the $T_{e} / T_{p}-V_{s}$ relation appears. The $T_{e} / T_{p}$ ratios for those cases are found to be $\sim 0.03$, superficially similar to $\sqrt{m_{e} / m_{p}}$, rather than $m_{e} / m_{p}$. The reason for the discrepancy is not clear, nor whether this indicates a breakdown in the $V_{s}^{-2}$ dependence at shock speeds exceeding $2000 \mathrm{~km} \mathrm{~s}^{-1}$. Some caveats to consider when interpreting the upturn in $T_{e} / T_{p}$ seen in Figure 2 are as follows: For shock speeds $\gtrsim 2000 \mathrm{~km} \mathrm{~s}^{-1}$ collisional ionization and excitation of $\mathrm{H}$ are primarily caused by proton (and to a lesser degree, alpha particle) impact (Laming et al. 1996; Ghavamian 1999; Ghavamian et al. 2001; Tseliakhovich et al. 2012). Experimentally measured cross sections for these interactions have still not been available to high precision (uncertainties $\sim 20 \%-30 \%$ still exist), although more sophisticated theoretical calculations are now becoming available (see, for example, Tseliakhovich et al. 2012) . In addition, as the broad component width increases, the $\mathrm{H} \alpha$ profiles are spread out over an increasing number of pixels, resulting in noisier spectra and greater measurement uncertainty in the broad component width. These larger error bars in turn result in a larger uncertainty in $V_{s}$, especially at shock speeds of $2000 \mathrm{~km} \mathrm{~s}^{-1}$ and higher.

The best way to further constrain the equilibration-shock speed relation is to add new data points to the curve shown in Figure 2 To this end, we present Balmerdominated $\mathrm{H} \alpha$ profiles for two additional positions in Tycho's SNR (different from those of Knot g presented by Kirshner, Winkler \& Chevalier (1987), Smith et al. (1991) and Ghavamian et al. (2001)). These profiles, shown in Figure 3, were acquired with a moderate resolution spectrograph in 1998 (for details on the observational setup, see Ghavamian 1999 and Ghavamian et al. 2001). The profile marked 'NE' was obtained from a clump of $\mathrm{H} \alpha$ emission located along the northeastern edge of Tycho's SNR, approximately $1^{\prime}$ northward of Knot $\mathrm{g}$. The clump appears behind the main body of 


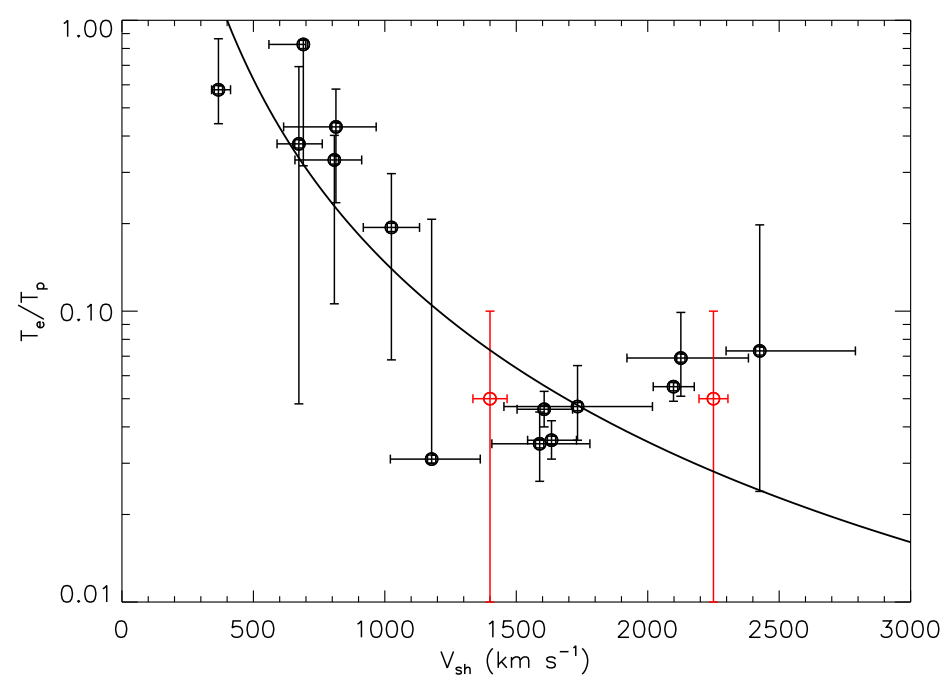

Fig. 2 The correlation between $\beta$ ( $\left.\equiv T_{e} / T_{p}\right)$ and shock speed for Balmer-dominated SNR shocks is shown, using results from van Adelsberg et al. (2008). The solid curve shows the $V_{S}^{-2}$ dependence inferred by Ghavamian et al. (2007) for Balmer-dominated SNR shocks. New results quoted from the NE and NW portions of Tycho's SNR (this paper) are marked in red. The apparent upturn at $V_{s} \gtrsim 2000 \mathrm{~km} \mathrm{~s}^{-1}$ and its associated error bars are discussed more fully in the text.

the Balmer filaments and exhibits a broad component that is substantially Doppler shifted to the red $\left(10.7 \pm 1.2 \AA\right.$, or about $\left.490 \mathrm{~km} \mathrm{~s}^{-1}\right)$. The Doppler shift reflects the bulk velocity of the hot posthock proton distribution, so the significant velocity shift of the broad component centroid indicates that the shock in the NE has a substantial velocity component into the plane of the sky, i.e., that the NE shock is located on the far side of the blast wave shell.

The broad $\mathrm{H} \alpha$ width of the NE shock is $1300 \pm 65 \mathrm{~km} \mathrm{~s}^{-1}$ (the smallest broad component width measured in Tycho's SNR so far), with $I_{b} / I_{n}=0.85 \pm 0.04$. The NW shock, on the other hand, has a broad $\mathrm{H} \alpha$ width of $2040 \pm 55 \mathrm{~km} \mathrm{~s}^{-1}$ (the largest broad component width measured in this SNR so far), with $I_{b} / I_{n}=0.45 \pm 0.15$. Neither of these broad-to-narrow ratios is strictly reproduced by the latest models of van Adelsberg et al. (2008), with the lowest predicted ratios being for Case B (the assumption of optically thick conditions for Ly $\beta$ photons in the narrow component) and for low equilibrations $\left(T_{e} / T_{p} \lesssim 0.1\right)$. For these low equilibrations, the corresponding shock speeds for the NE and NW shocks in Tycho are approximately $1400 \mathrm{~km} \mathrm{~s}^{-1}$ and 2250 $\mathrm{kms}^{-1}$ (using Figures 5 and 10 of van Adelsberg et al. 2008). In Figure 2 we have added data from the NE and NW shocks in Tycho's SNR to the $T_{e} / T_{p}-V_{s}$ plot. The two data points help fill in a portion of the plot where the data are sparse: the region between approximately $1200 \mathrm{~km} \mathrm{~s}^{-1}$ and $1500 \mathrm{~km} \mathrm{~s}^{-1}$, as well as the region beyond $2000 \mathrm{kms}^{-1}$, where the existing data are taken entirely from SN 1006. The added point near $1400 \mathrm{~km} \mathrm{~s}^{-1}$ is fully consistent with the $V_{s}^{-2}$ relation, while $T_{e} / T_{p}$ is not well constrained enough for the point at $2250 \mathrm{~km} \mathrm{~s}^{-1}$ to contradict the appearance of an upturn at the highest shock velocities. It is clear that proper characterization of the equilibration-shock velocity curve above $2000 \mathrm{~km} \mathrm{~s}^{-1}$ will require both higher 


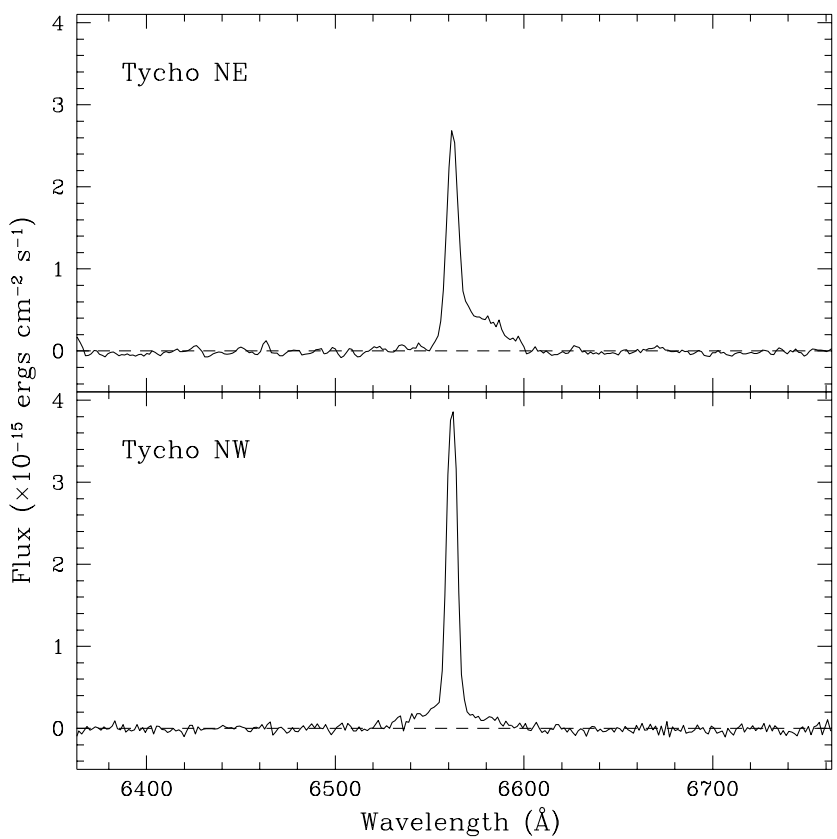

Fig. $3 \mathrm{H} \alpha$ profiles from two locations along the Balmer-dominated rim of Tycho's SNR (Ghavamian 1999). Top: the spectrum of a clump immediately to the north of Knot $g$ $\left(I_{b} / I_{n}=0.85 \pm 0.04 ; \mathrm{FWHM}=1300 \pm 65 \mathrm{~km} \mathrm{~s}^{-1}\right)$. The broad component is substantially redshifted from the center of the narrow component $\left(+10.7 \pm 1.2 \AA\right.$, or $\left.490 \pm 55 \mathrm{~km} \mathrm{~s}^{-1}\right)$, indicating that the clump is located on the far side of the shell, projected $\sim 20^{\circ}$ inside of the main Balmer-dominated rim. Bottom: profile acquired from the northernmost filament in Tycho, $I_{b} / I_{n}=0.45 \pm 0.15, \mathrm{FWHM}=2040 \pm 55 \mathrm{~km} \mathrm{~s}^{-1}$.

signal-to-noise spectra on existing Balmer-dominated shocks, as well as new data points beyond a broad component width of $2500 \mathrm{~km} \mathrm{~s}^{-1}$.

\subsection{Exceptions to $T_{e} / T_{p} \propto \mathrm{V}_{s h}^{-2}$}

Although the inverse squared relation between equilibration and shock velocity appears has been the most salient result of the study of Balmer-dominated shocks, there have been discrepant results reported in a small subset of cases. Recently the broad $\mathrm{H} \alpha$ component in the LMC SNR 0509-67.5 was detected for the first time (Helder, Kosenko \& Vink 2010). Broad emission was observed along both the northeastern rim (FWHM $3900 \pm 800 \mathrm{~km} \mathrm{~s}^{-1}$ ) and southwestern rim (FWHM $2680 \pm 70 \mathrm{~km} \mathrm{~s}^{-1}$ ), with the former being the fastest Balmer-dominated shock detected to date having the characteristic broad and narrow component $\mathrm{H} \alpha$ emission. Interestingly, the broad-to-narrow ratios for both shocks are exceptionally low, $I_{b} / I_{n}=0.08 \pm 0.02$ and $0.29 \pm 0.01$ along the NE and SW rims, respectively. As noted by Helder, Kosenko \& Vink (2010) these ratios are nearly twice as large as the smallest ones predicted by the models of van Adelsberg et al. (2008), precluding measurement of $T_{e} / T_{p}$ from the Balmer-dominated spectra and 
implicating excess narrow component $\mathrm{H} \alpha$ emission in a cosmic ray precursor once again. Using RGS spectra of SNR 0509-67.5 acquired with XMM-Newton, they obtained a forward shock speed of approximately $5000 \mathrm{~km} \mathrm{~s}^{-1}$ in the SW. This implies a broad $\mathrm{H} \alpha$ width of $3600 \mathrm{~km} \mathrm{~s}^{-1}$, substantially smaller than the measured width of 2680 $\mathrm{kms}^{-1}$. Helder, Kosenko \& Vink (2010) suggest that this indicates some thermal energy loss $(\sim 20 \%)$ to cosmic ray acceleration. This picture is supported by the presence of nonthermal X-ray emission in their fitted RGS spectra of SNR 0509-67.5.

The result described above relies upon an accurate disentangling of the bulk Doppler broadening from the thermal Doppler broadening in the X-ray lines. The disentangling depends on parameters such as the ratio of reverse shock to forward shock velocities, as well as the ratio of the gradients in the reverse and forward shock velocities, which in turn had to be assumed from evolutionary SNR models. The result, while intriguing, is still significantly uncertain. On the other hand, Helder, Kosenko \& Vink (2010) found that the X-ray shock velocities from the NE could only be reconciled with the observed broad $\mathrm{H} \alpha$ width there if $T_{e} / T_{p} \approx 0.2$, which clearly which predicts $T_{e} / T_{p} \sim m_{e} / m_{p}$ predicted from Equation 5 If this result were to be confimed by future observations, it would present a new challenge in understanding how electron-ion equilibration occurs in fast collisionless shocks. One possibility, given the presence of nonthermal X-ray emission in the spectra of 0509-67.5 (Warren \& Hughes 2004; Helder, Kosenko \& Vink 2010) is that the moderate loss of thermal energy to cosmic ray acceleration may have slightly increased the compression and reduced the temperature at the shock front compared to the case with no acceleration (Decourchelle, Ellison \& Ballet 2000; Ellison et al. 2007). Both of these effects would tend to render the plasma more collisional, possibly explaining the $T_{e} / T_{p} \approx 0.2$ result. However, it is also worth noting that the shock velocity used for obtaining $T_{e} / T_{p}$ in the NE is subject to the same model dependence and uncertainties as the SW measurements, so similar caution is required in its interpretation.

A similar combined optical and X-ray study of RCW 86 was performed by Helder et al. $(2009,2011)$. There, the broad component H $\alpha$ widths were supplemented with electron temperatures measured from XMM-Newton RGS spectra from the same projected locations along the rim. One of the main results of this study was that the slower shocks (broad H $\alpha$ FWHM $\sim 500-600 \mathrm{~km} \mathrm{~s}^{-1}$ ) showed showed $T_{e} / T_{p} \sim 1$, agreeing with earlier results from similar shocks observed both in RCW 86 and elsewhere (Ghavamian et al. (1999, 2001, 2007)). However, Helder et al. (2011) found while the results were indeed consistent with low equilibration at the shock front for fast shocks $\left(T_{e} / T_{p} \approx 0.02\right.$ for broad FWHM $\left.\left.\sim 1100 \mathrm{~km} \mathrm{~s}^{-1}\right)\right)$ and higher equilibration for the slower shocks $\left(T_{e} / T_{p} \approx 1\right.$ for broad FWHM $\left.\sim 650 \mathrm{~km} \mathrm{~s}^{-1}\right)$, their X-ray derived electron temperatures were inconsistent with $T_{e}=0.3 \mathrm{keV}$ at the shock front contradicting the suggestion of Ghavamian et al. (2007) that shocks above $400 \mathrm{~km} \mathrm{~s}^{-1}$ may all heat electrons to roughly $0.3 \mathrm{keV}$. However, a major caveat of these results is that the forward shock in RCW 86 is believed to be impacting the walls of a wind-blown bubble (Williams et al. 2011), resulting in substantial localized variations in shock speed around the rim. These variations occur as different parts of the forward shock impact the cavity wall at different times. While the broad component $\mathrm{H} \alpha$ widths closely trace the current position of the shock front, the X-ray emission behind that shock arises over a much more extended spatial scale, and is sensitive to the history of the forward shock interaction with the cavity wall. Furthermore, narrowband $\mathrm{H} \alpha$ imagery of RCW 86 with the ESO Very Large Telescope (Helder et al. (2009, 2011)) shows a complex morphology of filaments, especially along the eastern side of the SNR. The broad $\mathrm{H} \alpha$ 
components of these filaments exhibit substantial, localized variations in line width, ranging from $\sim 600 \mathrm{~km} \mathrm{~s}^{-1}$ FWHM to $1100 \mathrm{~km} \mathrm{~s}^{-1}$ Ghavamian (1999), Helder et al. $(2009,2011))$. These variations reflect localized changes in density and viewing geometry along the line of sight. As such, uniquely mapping the observed Balmer filaments to their corresponding X-ray emission in XMM-Newton data (especially given the somewhat coarse $10^{\prime \prime}$ spatial resolution of that instrument) is fraught with uncertainty. Additional corroboration for these results would be desirable.

\subsection{Imprint of the Shock Precursor on the $\mathrm{H} \alpha$ Line Profile}

Perhaps the most important and exciting recent development in our understanding of Balmer-dominated shocks has come with the development of new kinetic-based shock models. Blasi et al. (2012) have introduced a kinetic model for following the momentum and energy exchange between neutrals and ions, along with the back-reaction of those neutrals when they pass back upstream and form a fast neutral precursor. Rather than assume a Maxwellian velocity distribution for the neutrals (as had been done in previous models, despite the lack of thermal contact between neutrals needed to justify such an assumption), both the ion and neutral distributions are computed from their appropriate Boltzmann equations. Building on these models, Morlino et al. (2012a,b) have confirmed what had been suspected earlier (Smith et al. 1994; Hester et al. 1994; Sollerman et al. 2003), namely that the broadening of the narrow component beyond the expected ISM value $\left(\sim 25-30 \mathrm{~km} \mathrm{~s}^{-1}\right.$ instead of $\left.10 \mathrm{~km} \mathrm{~s}^{-1}\right)$ is most likey due to heating in a cosmic ray precursor. In particular, Morlino et al. (2012) found that the characteristic charge exchange length of the incoming neutrals exceeds that of the neutrals crossing back upstream, so that the narrow component width is impacted not by the neutral return flux, but rather by heating in the cosmic ray precursor. Raymond et al. (2011) predicted a similar broadening of the narrow component, though they focused mainly on the contribution of collisional excitation in the precursor to the flux in the narrow $\mathrm{H} \alpha$ component. Spatially resolved line broadening of the narrow $\mathrm{H} \alpha$ component was detected in ground-based longslit spectroscopy of Knot $\mathrm{g}$ in Tycho (Lee et al. 2007). In addition, a small ramp-up in $\mathrm{H} \alpha$ emission was observed ahead of Knot g in HST imagery Tycho's SNR (Lee et al. 2010). The results, taken together, are strong evidence for the presence of cosmic ray precursors in Balmer-dominated SNRs.

One prediction of the new Balmer-dominated shock models is the existence of a third component of the $\mathrm{H} \alpha$ emission (Morlino et al. 2012a,b). When the hot neutrals escape upstream, they undergo charge exchange with the colder preshock protons. This results in fast protons with cold neutrals, with the former rapidly equilibrating with preshock protons and pre-heating the gas. The temperature of the equilibrated protons in the precursor lies between the temperature of the far upstream protons $(\sim 5000 \mathrm{~K})$ and the far downstream protons $\left(\sim 10^{6}-10^{8} \mathrm{~K}\right)$, typically $\sim 10^{5} \mathrm{~K}$. Further charge exchange between these warm protons and the preshock neutrals gives rise to a third, 'warm' neutral component (neither fast nor slow) having velocity widths of hundreds of $\mathrm{km} \mathrm{s}^{-1}$ (Morlino et al. 2012a). Interestingly, the presence of a third $\mathrm{H} \alpha$ component was first observationally reported by Smith et al. (1994) in their high resolution echelle spectroscopy of Balmer-dominated SNRs in the Large Magellanic Cloud (an example of one of the spectra from Smith et al. (1994) is reproduced in Figure 44). A third $\mathrm{H} \alpha$ component was also reported in high resolution spectra of Knot $\mathrm{g}$ in Tycho's SNR (Ghavamian et al. 2000). In their models of Balmer-dominated shock emission, Morlino 


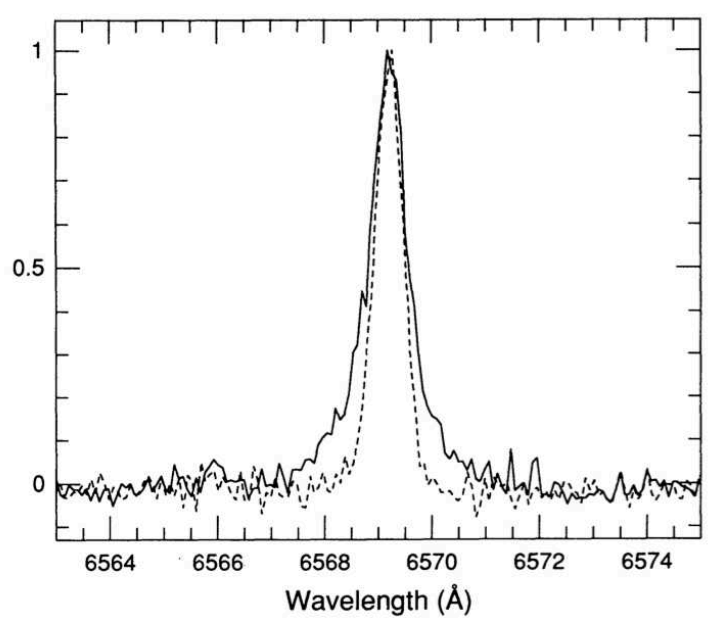

Fig. 4 High dispersion $\mathrm{H} \alpha$ profiles of the Balmer-dominated SNR 0509-67.5 in the Large Magellanic Cloud (Smith et al. 1994). The broad component is not detected due to the high dispersion. The narrow component from the east rim of the SNR (solid line) shows intermediate velocity wings of width $\sim 100 \mathrm{~km} \mathrm{~s}^{-1}$, while that of the center (dotted line) does not. The intermediate component is now believed to arise within a fast neutral precursor (Morlino et al. 2012a).

et al. (2012a) found that the importance of the third component relative to that of the broad and narrow components depends strongly on the preshock neutral fraction and $T_{e} / T_{p}$, in line with earlier theoretical predictions on properties of a fast neutral precursor (Smith et al. 1994). The fact that the third component has been detected in Tycho's SNR (width $\sim 150 \mathrm{~km} \mathrm{~s}^{-1}$ ) is consistent with the high preshock neutral fraction $\left(f_{H I} \sim 0.9\right)$ inferred from the broad-to-narrow ratio of Knot $\mathrm{g}$ by Ghavamian et al. (2001). A similar third component may have been detected in high resolution spectra of SNR 0509-67.5, where measurement of the narrow component width required the inclusion of an additiona component of width $75 \mathrm{~km} \mathrm{~s}^{-1}$ (Smith et al. 1994). The new fast neutral precursor models predict that a substantial fraction of the $\mathrm{H} \alpha$ excitation in Balmer-dominated shocks can arise ahead of the shock, where warm neutrals are excited by electron impact. Interestingly, the relative contribution of the preshock $\mathrm{H} \alpha$ to the total (upstream + downstream) is sensitive to $T_{e} / T_{p}$ behind the shock. Morlino et al. (2012a) found that up to $40 \%$ of the total $\mathrm{H} \alpha$ flux from a Balmer-dominated shock can arise from the fast neutral precursor when $V_{s} \sim 2500 \mathrm{~km} \mathrm{~s}^{-1}$ and $T_{e} / T_{p}=1$ both upstream and downstream. In these models the preshock contribution to the total flux drops substantially for lower downstream equilibrations for $V_{s} \gtrsim 1000 \mathrm{~km} \mathrm{~s}^{-1}$ (the slowest shocks considered by Morlino et al. 2012a). This is generally consistent with the fact that in most cases, shock models not including the precursor $\mathrm{H} \alpha$ emission have been able to match the observed broad-to-narrow ratios. In other words, if the postshock temperature equilibration were not low for such fast shocks, the agreement between the observed and predicted $I_{b} / I_{n}$ would have been substantially worse for such remnants as Tycho's SNR and SN 1006. 


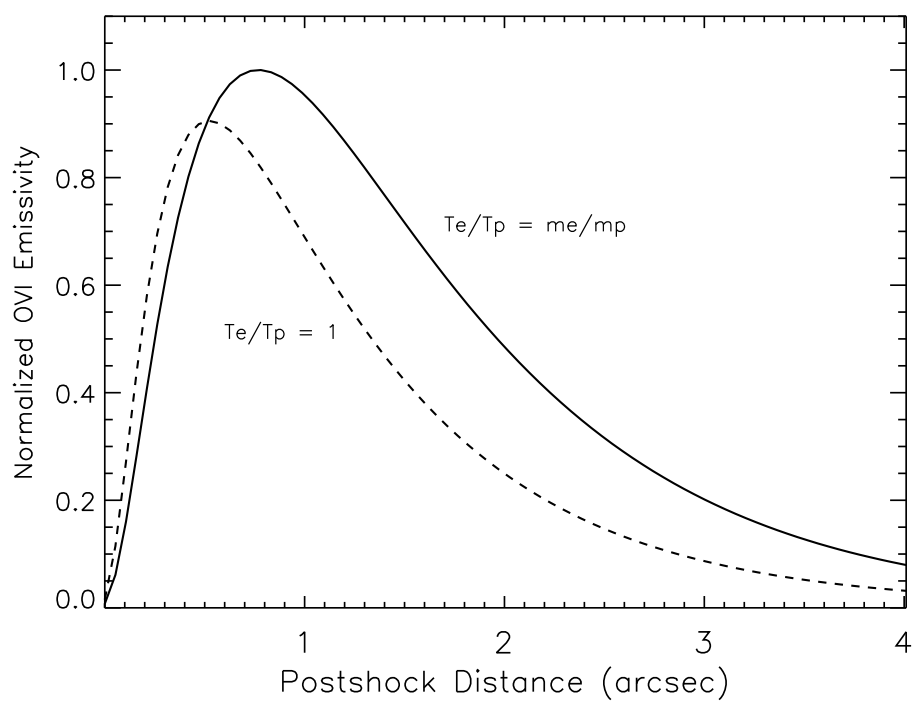

Fig. 5 The spatial distribution of the O VI(1032+1038) emissivity behind a $350 \mathrm{~km} \mathrm{~s}^{-1}$ planar shock at a distance of 500 pc (parameters appropriate to the fastest Balmer-dominated shocks in the Cygnus Loop), shown for the cases of full, instant equilibration at the shock front $\left(T_{e} / T_{p}=1\right)$ and minimal equilibration $\left(T_{e} / T_{p}=m_{e} / m_{p}\right)$. Analysis and modeling of the $\mathrm{H} \alpha$ profiles, as well as the $\mathrm{O}$ VI and X-ray emission indicate that $T_{e} / T_{p} \approx 1$ for the Cygnus Loop.

\subsection{Ultraviolet and X-ray Studies of Balmer-Dominated Shocks}

SN 1006 is an example of a SNR accessible to UV spectroscopy due to its galactic location, $450 \mathrm{pc}$ up from the galactic plane, and therefore with relatively low extinction due to intervening dust and gas. The Hopkins Ultraviolet Telescope (HUT) observed the UV resonance lines of He II $\lambda 1640$, C IV $\lambda \lambda 1548,1550$, N V $\lambda \lambda 1238,1243$, and O VI $\lambda 1032,1038$ (Raymond et al. 1995) emitted from the Balmer dominated filament in the NW quadrant. The line showed Doppler broadening consistent with that of the $\mathrm{H} \alpha$ broad component observed in the optical, indicating insignificant ion-ion equilibration. Laming et al. (1996) were also able to infer the degree of electron-ion equilibration at the shock.

He II $\lambda 1640$, by virtue of its relatively high excitation potential $\sim 48 \mathrm{eV}$ ), is excited only by electrons, and its intensity is therefore directly related to the electron temperature. C IV $\lambda \lambda 1548,1550, \mathrm{~N}$ V $\lambda \lambda 1238,1243$, and O VI $\lambda 1032,1038$, by contrast, have much lower excitation potentials of $\sim 8,10$ and $12 \mathrm{eV}$, so although these ionization states are established by electrons, the line emission in these transitions can also be excited by impacts with hot protons and $\alpha$ particles, and the intensity ratio of He II $\lambda 1640$ to C IV $\lambda \lambda 1548,1550, \mathrm{~N}$ V $\lambda \lambda 1238,1243$, and O VI $\lambda 1032,1038$ can be sensitive to the post-shock electron-proton temperature equilibration. The spatial 
distribution of the UV resonance line emission, when spatially resolved, provides additional constraints on the degree of electron-ion equilibration at the shock front. For shocks slower than $1500 \mathrm{kms}^{-1}, T_{e}=T_{p}$ at the shock front results in both a more rapid rise and higher maximum in emissivity of the UV resonance lines with distance behind the shock (see Figure 5 ).

Laming et al. (1996) calculated impact excitation cross sections for protons and $\alpha$ particles colliding with Li-like ions, using a partial wave expansion with the CoulombBethe approximation, and applying a unitarization procedure following Seaton (1964). They found a degree of equilibration of order $T_{e} / T_{p} \sim 0.05$ or less, which implied for a $2250 \mathrm{~km} \mathrm{~s}^{-1}$ shock an electron temperature immediately postshock of $<5 \times 10^{6} \mathrm{~K}$, in very good agreement (and actually predating) the optical results discussed above in subsection 4.1.

The ion-ion equilibration in SN 1006 was revisited by Korreck et al.(2004), using higher spectral resolution FUSE data comprising O VI $\lambda 1032,1038$ and the broad Ly $\beta \lambda 1025$ emission lines. They found a slightly broader line profile in Ly $\beta$, implying less than mass-proportional heating and possibly a small degree of ion-ion equilibration.

SN 1987A represents another SN/SNR in a region of the sky accessible to UV observations. HST COS observed the He II $\lambda 1640$, C IV $\lambda \lambda 1548$, 1550, N V $\lambda \lambda 1238,1243$ and N IV $\lambda \lambda 1486$ lines emitted from the reverse shock (France et al. 2011). When combined with optical spectroscopy of $\mathrm{H} \alpha$, the $T_{e} / T_{p}$ ratio at the shock is determined to be in the range $0.14-0.35$, significantly higher than similar ratios coming from Balmer dominated forward shocks. France et al. (2011) argued that a different equilibration mechanism is likely at work. Considering the relative youth of SN 1987A, and the fact that the reverse shock is the origin of the emission, significant populations of cosmic rays and associated magnetic field amplification are unlikely. In fact, in the expanding ejecta the magnetic field is likely to be very weak, leading to a very high Aflvén Mach number shock. As will be discussed below in connection with shocks in galaxy clusters, electron heating in such a case is likely to be due to acceleration in the cross-shock potential. The cross-shock potential is effective at heating electrons, and so may explain the higher $T_{e} / T_{p}$ in SN 1987A.

The forward shock of SN 1987A has also been observed in X-rays with the grating instruments on Chandra (e.g. Zhekov et al. 2009). In general electron heating well below complete equilibration is seen, though precise interpretation is difficult because one observation sees emission from shocks at a variety of different velocities, due to irregularities in the density of the surrounding medium.

3.5 Do Results from the Balmer-Dominated Shocks Apply to Fully Ionized Shocks?

The inverse relationship between the temperature equilibration and shock speed is an interesting result from studies of Balmer-dominated SNRs. However, the applicability of this result to both fully ionized shocks and shocks undergoing efficient CR acceleration ( $250 \%$ of their energy transferred to CRs) remains unsettled. Recently, Vink et al. (2010) used a two-fluid-model for cosmic rays and thermal gas to simulate the effect of cosmic ray acceleration on the temperature and ionization structure of fast, nonrelativistic shocks. They found that if $5 \%$ of the shock energy were to be channeled into cosmic rays (the minimum needed if SNRs are the dominant source of cosmic rays) then approximately $30 \%$ of the postshock pressure must reside in cosmic rays (corresponding to a ratio of cosmic ray to total postshock pressure, $w$, of 0.3 ). For $w=0.3$, Vink et al. 
(2010) predicted a lowering of the average temperature of the postshock gas to $\sim 70 \%$ of the value when the cosmic ray contribution is ignored. This is a significant alteration of the postshock temperature profile, and should result in much more rapid equilibration of electrons and protons close to the shock.

However, do the effects described above actually occur in SNR shocks? One of the principle lines of evidence cited by Vink et al. (2010) in support of this picture was the result found in RCW 86 by Helder et al. (2009). In that SNR, the broad $\mathrm{H} \alpha$ widths of Balmer-dominated filaments were found to be nearly $50 \%$ smaller than the minimum allowed given their X-ray proper motions. Filaments in the NE of the SNR exhibited broad $\mathrm{H} \alpha$ widths of $1000 \mathrm{~km} \mathrm{~s}^{-1}$, but their apparent X-ray counterparts, which exhibited strong X-ray synchrotron (nonthermal) emission, exhibited proper motions indicating shock speeds of $3000-6000 \mathrm{~km} \mathrm{~s}^{-1}$. This result, along with the theoretical prediction that X-ray synchrotron emission requires shock speeds of at least $2000 \mathrm{~km} \mathrm{~s}^{-1}$ (Aharonian et al. 1999) was taken by Helder et al. (2009) and Vink et al. (2010) as evidence for substantial energy loss $(\mathrm{w} \sim 0.5)$ from the Balmerdominated shocks to cosmic rays. However, this association has now been refuted by subsequent multi-epoch optical imagery of the $\mathrm{H} \alpha$ filaments, which have failed to show the kind of high proper motions seen in the nonthermal X-ray filaments (Helder et al. 2012, in preparation). Instead, they show proper motions consistent with shock speeds predicted by the broad $\mathrm{H} \alpha$ widths without energy loss to cosmic rays $(\sim 600-$ $1200 \mathrm{~km} \mathrm{~s}^{-1}$ ), implying that $\mathrm{w}<0.2$. The association between the Balmer-dominated shocks studied spectroscopically by Helder et al. (2009) and the X-ray filaments was due either to coincidental spatial alignment, or due to sudden deceleration of the outer shock in RCW 86 during its encounter with the surrounding cavity wall (Williams et al. 2011; Helder et al. 2012, in preparation).

The lack of association between the Balmer-dominated filaments and the nonthermal X-ray filaments in RCW 86 raises some important questions about the feasibility of using Balmer-dominated shocks to study electron-proton equilibration in cases where $50 \%$ or more of the thermal energy is diverted to cosmic rays. In SNRs such as SN 1006 (Koyama et al. 1996; Katsuda et al. 2010a), Tycho's SNR (Warren et al. 2005; Katsuda et al. 2010b), Cas A (Vink \& Laming 2003) and RX J1713.7-3946 (Koyama et al. 1997; Slane et al. 1999; Tanaka et al. 2008) the presence of strong synchrotron X-ray filaments has been interpreted as evidence for highly efficient cosmic ray acceleration. The narrowness of the synchrotron filaments most likely reflects the short emitting lifetimes of the ultra high energy electrons (energies $\sim 10-100 \mathrm{TeV}$ ) as they spiral in the postshock magnetic field (Vink \& Laming 2003). The detection of $\gamma$-ray emission from the shells of SN 1006 (Acero et al. 2010) and RX J1713.7-3946 (Aharonian et al. 2006; Abdo et al. 2011) has shown that cosmic rays are accelerated to energies as high as $100 \mathrm{TeV}$ in these SNRs. In all cases thermal X-ray emission has been exceptionally faint due to the very low inferred preshock densities $\left(\lesssim 0.1 \mathrm{~cm}^{-3}\right)$, making it more likely that the overall X-ray emission will be dominated by synchrotron radiation from the most energetic cosmic rays. SNRs expanding into such low density media can propagate at the high shock speeds required for cosmic ray acceleration $\left(\gtrsim 2000 \mathrm{~km} \mathrm{~s}^{-1}\right.$; Aharonian et al. 1999) for a longer time, allowing their structure to become modified by the back pressure from the cosmic rays. Investigating the temperature and ionization structure of such shocks with Balmer line spectroscopy requires finding Balmer-dominated shocks exhibiting X-ray synchrotron radiation.

All of the known SNRs exhibiting Balmer-dominated shocks have also been imaged at X-ray wavelengths with Chandra or XMM, allowing reasonably detailed searches for 


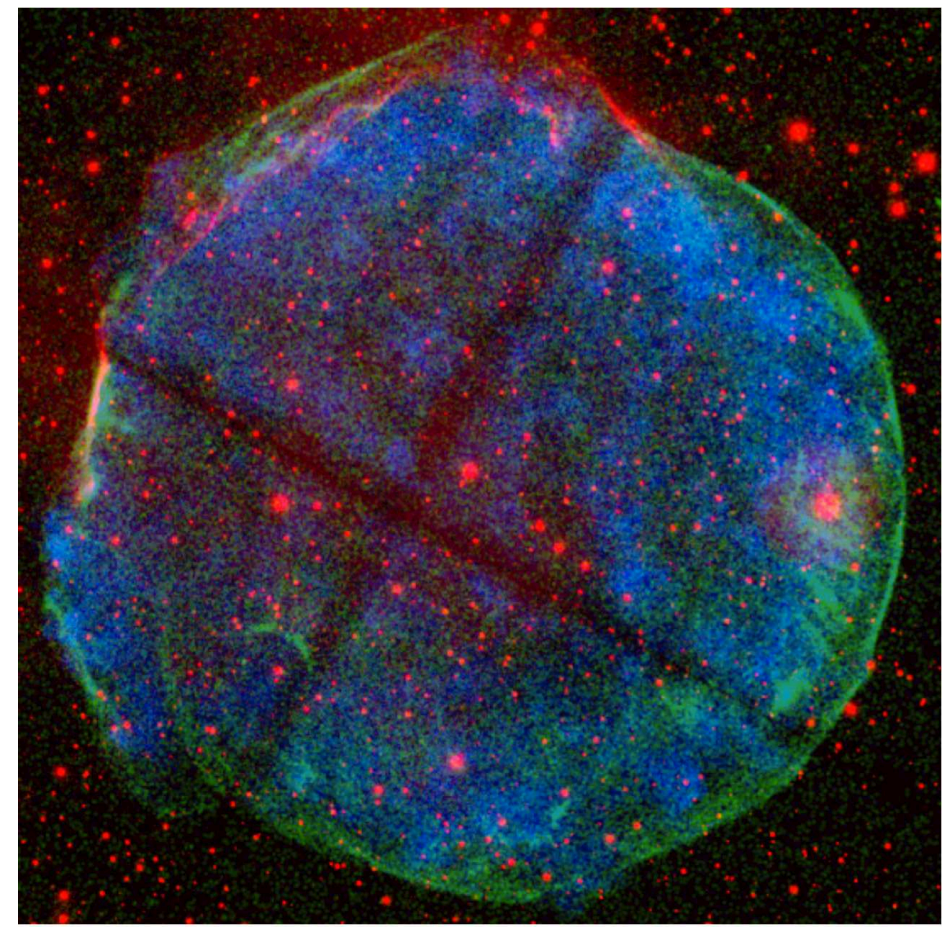

Fig. 6 An H $\alpha$ narrowband image of Tycho's SNR (red; courtesy P. F. Winkler), overlaid onto the Chandra 3-8 keV hard X-rays (green) and Chandra 0.5-3 keV soft X-rays (blue). Both optical and X-ray images were acquired during the same epoch (2007). Note the near mutual exclusivity of the Balmer emission and hard X-ray emission.

shocks emitting both $\mathrm{H} \alpha$ and synchrotron X-ray emission (the latter producing hard continuum that is dominant at energies of $2 \mathrm{keV}$ and higher). A detailed comparison for all Balmer-dominated SNRs has not yet been published. However, even a cursory comparison between the narrowband $\mathrm{H} \alpha$ and hard X-ray images of these SNRs shows a distinct anticorrelation between shocks emitting in these two bands. For example, overlaying $\mathrm{H} \alpha$ and Chandra images of Tycho's SNR acquired during the same epoch (2007) (Figure 6) shows little or no correlation between the prominent Balmer-dominated fil- 
aments on the eastern and northeastern edges and the non-thermal X-ray filaments $(\mathrm{E} \geq 3 \mathrm{keV})$ circling the remnant. The Balmer-dominated filaments (shown in red in Figure 6) on the eastern side of Tycho's SNR are seen projected $30^{\prime \prime}-1^{\prime}$ inside the edge of the nonthermal X-ray filaments (marked in green), an indication that portion of the shell along the line of sight has significantly decelerated. The Balmer filaments are seen at the outermost edge of the thermally emitting X-ray ejecta (marked in blue), but only at locations where little or no nonthermal X-ray emission is present. The bright optical filament known as Knot $\mathrm{g}$ (at the far left edge of Figure 6) is the only location where Balmer filaments and X-ray synchrotron emission appear coincident. However, upon closer inspection the anticorrelation between the Balmer line and synchrotron emission can be seen in Knot $\mathrm{g}$ as well: the upper half of the filament, where Balmer line emission is strongest, exhibits minimal synchrotron emission, while the opposite is true in the lower half of the filament. The enhanced nonthermal emission inside of Knot g may be due to the strong recent deceleration of Knot g, where the SNR is currently propagating into a strong density gradient at the outermost edge of an $\mathrm{H}$ I cloud (Ghavamian et al. 2000). The lack of optical/X-ray synchrotron correlation is especially striking given that the Balmer-dominated filaments in Tycho's SNR have a high enough shock velocity $\left(\sim 1800-2100 \mathrm{~km} \mathrm{~s}^{-1}\right)$ to accelerate particles to $\mathrm{TeV}$ energies.

The anticorrelation between the optical and nonthermal X-ray emission can be observed in other SNRs as well, including SN 1006, where recently X-ray proper motions have been measured along the entire rim by Katsuda et al. (2012). As with Tycho's SNR, the locations of the Balmer-dominated filaments and the nonthermal X-ray filaments along the NW rim of SN 1006 are mutually exclusive. Instead, the Balmer-dominated shocks are closely associated with thermal X-ray filaments having a proper motion consistent with a shock velocity of $3300 \pm 200 \pm 300 \mathrm{~km} \mathrm{~s}^{-1}$ (statistical and registrational uncertainties, respectively) for a distance of $2.2 \mathrm{kpc}$. This result is in excellent agreement with the shock velocity of $2890 \pm 100 \mathrm{~km} \mathrm{~s}^{-1}$ determined from the broad $\mathrm{H} \alpha$ width and broad-to-narrow ratio of the NW filament by Ghavamian et al. (2002). Such close agreement is a strong indication that little substantial energy has been lost from the thermal plasma to cosmic ray acceleration, similar to optical proper motion studies from RCW 86 (Helder et al., in preparation).

From the above discussion it appears that Balmer-dominated SNRs, while offering powerful diagnostics of $T_{e} / T_{p}$ and $V_{s}$, are not useful for investigating equilibration the extreme cases of strongly cosmic-ray modified shocks. In fact, the very condition allowing for the detection of the Balmer line emission - presence of neutral gas ahead of the shock - is also responsible for limiting the fraction of shock energy lost to cosmic ray acceleration. Quantatitive evaluations of this effect by Drury et al. (1996) and Reville et al. (2007) show that when the preshock gas is significantly neutral, Alfvén waves driven by the cosmic rays ahead of the shock are dissipated by ion-neutral damping. As long as the charge exchange frequency, $\omega_{c x}\left(\equiv n_{H I}\left\langle\sigma_{c x} v\right\rangle\right)$ is larger than the Alfvén wave frequency, $\omega_{A}\left(\equiv k v_{A}\right)$ the ions and neutrals oscillate coherently, and ion-neutral damping is not important. However, when $\omega_{c x}<\omega_{A}$ the neutrals are left behind by the ions in the Alfvén wave motion, and during the incoherent oscillation between the two, charge exchange exerts a drag on the Alfvén waves, damping them. The condition required for Alfvén waves to not be strongly damped in the precursor can be written out as

$$
n_{H I}\left\langle\sigma_{c x} v\right\rangle>k v_{A}
$$


where $v_{A} \equiv \frac{B}{\left(4 \pi m_{i} n_{i}\right)^{1 / 2}}$ is the Alfvén speed of the ions ahead of the shock and $n_{H I}$ is the preshock neutral density. Given that cosmic rays resonantly scatter off Alfvén waves having Doppler shifted frequencies comparable to their gyrofrequency, and that the cosmic ray gyrofrequency is related to its energy via $\omega_{c r} \equiv e c B / E$, the inequality above can be cast in terms of the energy, $E_{\text {crit }}$, below which a significant fraction of the cosmic ray flux out of the shock is reduced by ion-neutral damping:

$$
E_{\text {crit }}(T e V)=0.07 \frac{B_{3}^{2} T_{4}^{-0.4}}{x_{H I}\left(1-x_{H I}\right)^{1 / 2} n^{3 / 2}}
$$

where we have set $\left\langle\sigma_{c x} v\right\rangle \approx 8.4 \times 10^{-9} T_{4}^{0.4} \mathrm{~cm}^{3} \mathrm{~s}^{-1}$ (Kulsrud \& Cesarsky 1971), $B_{3}$ is the preshock magnetic field strength in units of $3 \mu G, n$ and $x_{H I}$ are the total preshock density and neutral fraction, and $T_{4}$ is the preshock temperature in units of $10^{4} \mathrm{~K}$. For Balmer-dominated SNRs, where recent models have required moderate amplification of the preshock magnetic field ( $\Delta B / B \sim 3-5$; Ghavamian et al. 2007) and where the preshock temperature may exceed 20,000 K (Raymond et al. 2011), $E_{\text {crit }} \sim 4$ $\mathrm{TeV}$ for the typical case where $x_{H I}=0.5 \mathrm{~cm}^{-3}$. SNRs exhibiting nonthermal X-ray emission are believed to contain cosmic rays with energies of tens of $\mathrm{TeV}$, so $E_{\text {crit }} \sim 4$ $\mathrm{TeV}$ is certainly high enough reduce the effectiveness of Balmer-dominated shocks in producing nonthermal X-ray emission.

However, as noted earlier, a modest back pressure from cosmic rays is required to explain the width of the $\mathrm{H} \alpha$ narrow component line, as well as the low broad-to-narrow ratios seen in some SNRs (Rakowski et al. 2009; Raymond et al. 2011). In fact, one model for electron heating in fast collisionless shocks requires at least some feedback from the cosmic rays in order to explain the moderate heating of electrons in SNRs, as well as the inverse squared relationship between $T_{e} / T_{p}$ and $V_{s}$ (Ghavamian et al. 2007, described in the next section). Furthermore, as pointed out by Drury et al. (1996), the ion-neutral damping of Alfvén waves in the precursor is unimportant for cosmic rays which have already exceeded $E_{\text {crit }}$. Since the acceleration time for cosmic rays shortens considerably with shock speed $\left(\tau_{a c c} \approx \kappa_{C R} / V_{s}^{2}\right.$; Malkov \& Drury 2001), the fastest Balmer-dominated shocks are more likely to have accelerated particles beyond $E_{\text {crit }}$ and hence will begin to exhibit nonthermal X-ray emission and cosmic-ray modified shock structure. A good example is the aforementioned SNR 0509-67.5, where the shock speeds exceed $5000 \mathrm{~km} \mathrm{~s}^{-1}$ (Helder, Kosenko \& Vink 2010) and nonthermal $\mathrm{X}$-ray emission from cosmic ray accelerated electrons is detected from the forward shock. The forward shocks in more evolved Balmer-dominated SNRs (such as SN 1006 and Tycho's SNR) will have swept up more mass and slowed down to speeds $\lesssim 2000$ $\mathrm{km} \mathrm{s}^{-1}$, by which point $\tau_{a c c}$ will have lengthened and the shocks will be less cosmic-ray dominated.

\section{Models for Electron Heating in SNRs}

Given the lack of in situ measurements of the particle distributions in SNRs, the electron heating mechanisms in these shocks have been studied primarily via numerical methods. On one hand, a number of studies have focused on electron heating in relativistic shocks, with the aim of modeling high energy emission from gamma-ray bursts (e.g., Gedalin et al. 2008; Sironi \& Spitkovsky 2011). These shocks are in a different area of parameter space than the SNR shocks discussed here, and the physics governing 
the electron heating in relativistic shocks is substantially different. At the very high Alfvénic Mach numbers characteristic of gamma-ray bursts, the shock transition becomes very thin (less than an electron gyroradius). Electrons in this case may be once again accelerated by the cross shock potential, similar to the very low Mach number case. On the other hand, a number of other studies consider non-relativistic shocks relevant to SNRs $(\lesssim 0.01 \mathrm{c})$, where accelerated particles such as cosmic rays or solar energetic particles (SEPs) may play an important role in establishing their shock structure. These studies have sought to identify plasma waves capable of boosting electrons to mildly relativistic energies (e.g., Amano \& Hoshino 2010; Riquelme \& Spitkovsky 2011), with the objective of understanding how electrons are injected into the cosmic ray acceleration process. This is a different (though related) question from what we consider here, namely how electrons are promptly heated to temperatures $\sim 5 \times 10^{6} \mathrm{~K}$ at the shock front (Ghavamian et al. 2007; Rakowski et al. 2008). This limits our consideration of the work done so far to two broad scenarios of electron heating in fast, non-relativistic collisionless shocks. One scenario is based on lower hybrid wave heating in the cosmic ray precursor (Laming 2000; Ghavamian et al. 2007; Rakowski et al. 2008) while the other is based on counterstreaming instabilities ahead of the shock (e.g., the Buneman instability, Cargill \& Papadapoulos (1988), Matsukiyo 2010; Dieckmann et al. 2012). We discuss these mechanisms below in turn.

\subsection{Lower Hybrid Wave Heating}

The most significant result of the Balmer-dominated shock studies, the inverse squared relation between $T_{e} / T_{p}$ and $V_{s}$, places a useful constraint on the range of plausible equilibration mechanisms at the shock front. The simplest way to obtain $T_{e} / T_{p} \propto V_{s}^{-2}$ is to set $\Delta T_{e} \approx$ const. at shock speeds of $400 \mathrm{~km} \mathrm{~s}^{-1}$ and higher, while allowing $T_{p}$ to rise according to the Rankine-Hugoniot jump conditions, $k \Delta T_{p} \approx \frac{3}{16} m_{p} V_{s}^{2}$. The requirement that $T_{e} / T_{p}=1$ at $V_{s}=400 \mathrm{~km} \mathrm{~s}^{-1}$ gives $\Delta T_{e} \approx 0.3 \mathrm{keV}$ for $V_{s} \geq 400$ $\mathrm{km} \mathrm{s}^{-1}$, independent of shock velocity (Ghavamian et al. 2007). Although there may be marginal evidence of a departure from this relation at shock speeds exceeding 2000 $\mathrm{km} \mathrm{s}^{-1}$ (van Adelsberg et al. 2008), a velocity-independent heating of electrons in SNR shocks is an important clue to the nature of plasma heating processes in fast collisionless shocks. It suggests that plasma processes ahead of the shock front are an important (if not dominant) source of electron heating in SNRs (Ghavamian et al. 2007; Rakowski et al. 2009).

As mentioned earlier, strong interstellar shocks are expected to form a precursor where cosmic rays crossing upstream give rise to Alfvén waves and turbulence (Blandford \& Eichler 1987; Jones \& Ellison 1991), compressing and pre-heating the gas before it enters the shock. As long as the shock is strong $\left(v_{\text {downstream }} / V_{s} \approx 1 / 4\right)$ and cosmic ray pressure does not dominate the postshock pressure $(\Delta \mathrm{B} / \mathrm{B}$ does not greatly exceed unity, with $\lesssim 20 \%$ of the postshock pressure provided by cosmic rays) the thermal heating within the precursor does not depend strongly on shock velocity. The limited range of narrow component $\mathrm{H} \alpha$ widths observed in Balmer-dominated SNRs over a wide range in shock speeds (Sollerman et al. 2003; Raymond et al. 2011) is consistent with the relative insensitivity of the preshock heating to shock speed. Since the widening of the $\mathrm{H} \alpha$ narrow component line is now believed to arise in a precursor where the gas is heated by the damping of cosmic-ray driven waves (Wagner et al. 2008; Raymond et al. 2011; Morlino et al. 2012), it stands to reason that perhaps the physical 
processes generating a constant electron heating with shock speed $\left(\Delta T_{e} \approx 0.3 \mathrm{keV}\right)$ also originate within the cosmic ray precursor.

The above argument was used by Ghavamian et al. (2007) and Rakowski et al. (2008) to advocate for a heating model where lower hybrid waves within the cosmic ray precursor preheat electrons to a constant temperature before they enter the shock front. This model was based on the conception of McClements et al. (1997), who suggested that lower hybrid waves driven by the reflected population of nonthermal ions could generate lower hybrid waves ahead of the shock, pre-heating electrons and injecting them into the cosmic ray acceleration process. The condition for generating such waves is that the shock be quasi-perpendicular, and that the reflected ions form a beam-like (gyrotropic) distibution. A similar scenario was suggested by Ghavamian et al. (2007) and Rakowski et al. (2008), but with one crucial difference: the reflected particles considered are ultra-relativistic cosmic rays rather than suprathermal ions. The lower hybrid waves are electrostatic ion waves which propagate perpendicular to the magnetic field and whose frequency is the geometric mean of the electron and ion geofrequencies, $\omega_{L H}=\left(\Omega_{e} \Omega_{i}\right)^{1 / 2}$. The group velocity of these waves is directed primarily along the magnetic field lines $\left(k_{\|}^{2} / k_{\perp}^{2}=m_{e} / m_{p}\right.$; Laming 2001) and the waves can simultaneously resonate with ions moving across the field lines and electrons moving along the field lines. Although the growth rate of lower hybrid waves is generally small (Rakowski et al. 2008), their group velocity perpendicular to the magnetic field (and hence the shock front) can be on the order of the shock velocity $\left(\partial \omega / \partial k_{\perp} \approx V_{s}\right)$. This allows the lower hybrid waves to remain in contact with the shock for long periods of time, attaining high intensities capable of effectively heating the electrons (McClements et al. 1997; Ghavamian et al. 2007).

In the case of cosmic rays, the time spent by the electrons in the precursor is $t \sim \kappa_{C R} / v_{s h}^{2}$. The kinetic energy acquired by the electrons in the precursor is $\Delta E_{e} \propto$ $D_{|| \mid} t$, where $D_{|| \mid}$is the momentum diffusion coefficient of electrons (Ghavamian et al. 2007). For lower hybrid wave turbulence, $D_{\|\| \|} \propto V_{s}^{2}$ (Karney 1978; Ghavamian et al. 2007; Rakowski et al. 2008), so that $\Delta E_{e} \approx \frac{1}{16}\left(\frac{m_{e}}{m_{p}}\right)^{1 / 2} m_{e} \Omega_{e} \kappa_{C R} \propto B \kappa_{C R} \sim$ const, as needed to account for the inverse squared relationship between equilibration and shock speed. Note that under the assumption that nonlinear amplification of the preshock magnetic field is not too strong $(\Delta B / B \sim 1), \kappa_{C R}$ is that of Bohm diffusion, which scales as $1 / \mathrm{B}$, so that $\Delta E_{e}$ is also approximately independent of $\mathrm{B}$.

During the past decade more refined models of cosmic ray acceleration have shown that a non-resonant mode of Alfvén waves, having a higher growth rate than the previously considered resonant mode (Skilling 1975), can be excited by cosmic rays in the precursor (Bell \& Lucek 2001, Bell 2004, 2005). Unlike for the resonant case, the non-resonant amplification allows for $\Delta B / B \gg 1$, driving preshock magnetic fields to values as high as $1 \mathrm{mG}$ (Vink \& Laming 2003; Berezhko et al. 2003; Bamba et al. 2005; Ballet 2006). Such magnetic fields are hundreds of times stronger than the canonical preshock magnetic field of $3 \mu G$ and high enough to account for the observed narrowness of X-ray synchrotron-emitting rims in such SNRs as SN 1006 (assuming the narrowness is due to rapid cooling of high energy electrons behind the shock; see Ballet 2006 and Morlino et al. 2012). Additional studies have suggested that non-resonant amplification may dominate early in the life of the SNR, while resonant amplification may take over during the Sedov-Taylor stage of evolution (Amato \& Blasi 2009; Schure et al. 2012), though in either case, $\Delta B / B>10$ is readily attained. Such a strong magnetic field effectively reduces the acceleration time for particles, and is 
very well suited for explaining how cosmic rays can reach the knee in the cosmic ray spectrum near $10^{15} \mathrm{eV}$ (Bell \& Lucek 2001; Eriksen et al. 2011).

An important factor influencing the effectiveness of lower hybrid wave heating of electrons is the orientation of the preshock magnetic field relative to the shock front. Lower hybrid wave heating is only effective in perpendicular shocks (Vink \& Laming 2003, Ghavamian et al. 2007; Rakowski et al. 2008). Given their spherical global geometry, SNR blast waves generally propagate at a range of angles to the interstellar magnetic field. X-ray observations and models of such SNRs as SN 1006 (Orlando et al. 2007; Petruk et al. 2008) have indicated that perpendicular shocks are far more effective at accelerating cosmic rays than parallel shocks. Although the detailed implications of such differences have not yet been worked out for the lower hybrid wave heating model, Rakowski et al. (2008) argue that even for quasi-parallel shock geometries the cosmic ray current driving the nonresonant Alfvén waves will generate a significant perpendicular magnetic field ahead of the shock (such a possibility has also been inferred from numerical simulations; Riquelme \& Spitkovsky 2011). This would allow lower hybrid wave growth to overtake modified Alfvén wave growth for arbitrary orientations of the far upstream magnetic field, and allow for a more ubiquitous role for lower hybrid wave heating of electrons.

The amplification of the preshock magnetic field well beyond its far upstream value introduces an interesting possibility: effective lowering of the Alfvénic (and hence magnetosonic) Mach number of the shock. For the Balmer-dominated shocks, where analysis of the optical spectra has shown that at best only a moderate fraction $(\lesssim 20 \%)$ of the shock energy has likely been channeled into cosmic rays, the widening of the $\mathrm{H} \alpha$ narrow component has been interpreted as nonthermal broadening caused by the lowest frequency waves in the precursor (Ghavamian et al. 2007; though see Raymond et al (2011) for a thermal intepretation). To explain the $30-50 \mathrm{~km} \mathrm{~s}^{-1}$ widths of the $\mathrm{H} \alpha$ narrow component, the preshock magnetic field must be enhanced by a factor of a few. For the non-resonant Alfvén waves in the Bell (2004) mechanism, the magnetic field energy density immediately behind the shock is given by (Schure et al. 2012)

$$
\frac{B^{2}}{4 \pi} \approx \frac{1}{4} \phi^{2} \rho v_{s h}^{2}
$$

where $\phi \equiv P_{C R} / \rho v_{s h}^{2}$ is the fraction of the shock ram pressure channeled into cosmic rays. Solving this expression for $\mathrm{B}$ gives $B(\mu G) \approx 228.7 \phi n^{1 / 2} V_{1000}$, where $V_{1000}$ is the shock speed in units of $1000 \mathrm{~km} \mathrm{~s}^{-1}$. For $\phi \sim 0.1-0.2, n \sim 1 \mathrm{~cm}^{-3}$, a postshock compression factor of 4 and Balmer-dominated shock speeds $\sim 2000 \mathrm{~km} \mathrm{~s}^{-1}$, this gives $\Delta B / B \sim 4-10$ ahead of the shock. Correspondingly, $v_{A}$ can increase by nearly the same factor, so that $M_{A}$ can be reduced by as much as an order of magnitude. Treumann \& Jaroschek (2008) describe the physical picture in this case as that of the shock having to prevent an increasing number of ions from crossing the shock jump by deflecting an increasing number of them at higher and higher Mach numbers. This deflection is necessary so that the ability of the shock to dissipate the inflowing energy is not overwhelmed. By deflecting these ions back upstream into a precursor, the net inflow of momentum and energy density into the shock is reduced, reducing the net difference in velocity between the inflowing and outflowing ions. This effectively reduces the Mach number in the frame of the upstream medium.

Note that the ions in the precursor are only mildly compressed (Wagner et al. 2008; Morlino et al. 2012), which only weakly counteracts the rise in B. In addition, $v_{A}$ only scales as $n^{-1 / 2}$, but scales directly as $\mathrm{B}$. The result is that given the compelling 
evidence for enhanced preshock magnetic fields in SNRs shocks, the Mach numbers of these shocks may be overestimated by as much as an order of magnitude. As we describe in Section 4, a unified description of solar wind and SNR shocks, where the physics of electron-ion temperature equilibration occurs over a similar range in Mach numbers and involves a similar range of physical processes, may be possible.

\subsection{Plasma Wave Heating from the Buneman Instability}

Similar to the lower hybrid wave model, the Buneman instability-driven wave model focuses on the region immediately ahead of a quasi-perpendicular shock. However, unlike the lower hybrid wave model, the Buneman instability models consider the reflected nonthermal ion distribution, rather than ultrarelativistic cosmic rays. In the latter model, $\sim 20 \%$ of the ions are reflected backstream against the incoming electron and ion plasma (Papadapoulos 1988; Cargill \& Papadapoulos 1988). Here the upstream plasma is not electrically neutral due to the positive charge of the reflected ion distribution. In such cases, a drift is induced between the electrons and ions. The microinstabilities excited by this configuration depend upon the relative size of the electron thermal speed relative to the electron-ion drift velocity. The Buneman instability occurs when the drift velocity of the reflected ions relative to the upstream electrons exceeds the thermal speed of the upstream electrons $\left(2 v_{s}>\left(k T_{e} / m_{e}\right)^{1 / 2}\right)($ Cargill \& Papadapoulos 1988), a condition which occurs for very high Mach number $\left(M_{A} \gtrsim 50\right)$ shocks. If the reflected ion current upstream is strong enough, the electron current generated to counteract it may produce a large enough drift between the preshock ions and electrons to cause a secondary Buneman instability when the ion speed exceeds the electron thermal speed (Dieckmann et al. 2012). The Buneman instability generates electrostatic plasma waves which damp by rapidly heating the preshock electrons to $k \Delta T_{e} \approx 2 m_{e} v_{s}^{2} \approx 0.01 v_{1000}^{2} \mathrm{keV}$, where $v_{1000}$ is the shock speed in units of $1000 \mathrm{~km} \mathrm{~s}^{-1}$. until their thermal speed matches the electron-ion drift speed, at which point the instability saturates. The rapid heating of the electrons perpendicular to the magnetic field results in $T_{e} / T_{i} \gg 1$ and makes it possible for an ion acoustic instability to occur between the preshock electrons and either the reflected ions or preshock ions (Cargill \& Papdapoulos 1988). The waves generated by the ion acoustic instability can then transfer a substantial fraction of the shock energy (tens of percent) into electron thermal energy. This process occurs over a length scale of $v_{s} / \Omega_{i}$ (as opposed to $\kappa_{C R} / v_{s}$ for the cosmic ray precursor), resulting in a $T_{e} / T_{p} \approx 0.2$, independent of shock speed. This is in strong disagreement with the equilibrations obtained for the Balmer-dominated shocks.

A number of other electron heating mechanisms, such as the modified two-stream instability and electron-cyclotron drift instability, have been proposed for collisionless shocks based on results from particle in cell (PIC) simulations (Umeda et al. 2012a, 2012b). A unified picture proposed by Matsukiyo (2010) suggests that electrons can be strongly energized at low Mach numbers $\left(M_{A} \leq 10\right)$ via a modified two-stream instability, where the velocities of the reflected/incoming ions and the electrons are lower than the thermal speed of the electrons and the electrons are able to damp out the Buneman instability. In this case, obliquely propagating whistler mode waves are excited, having frequencies between the electron cyclotron frequency and the lower hybrid wave frequency. When the electron-ion drift velocity and electron thermal speed become nearly equal, the electron-cyclotron drift instability becomes important (Umeda et al. 2012a), 
exciting waves with frequencies that are multiples of the electron cyclotron frequency. At higher Mach numbers the electron thermal speed is lower than that of the ions, and the Buneman instability/ion acoustic wave process descrbied earlier is predicted to take over.

The amount of electron heating predicted by the Buneman instability/ion acoustic wave model scales as $M_{A}^{2}$ (Cargill \& Papadapoulos 1988; Matsukiyo 2010), so that for shocks in the $2000 \mathrm{~km} \mathrm{~s}^{-1}-10,000 \mathrm{~km} \mathrm{~s}^{-1}$ range, $\Delta E_{e} \sim 2-50 \mathrm{keV}$. This is clearly at odds with $\Delta E_{e}=0.3 \mathrm{keV}$ observed between $400 \mathrm{kms}^{-1}$ and $2000 \mathrm{~km} \mathrm{~s}^{-1}$ for Balmer-dominated shocks. One explanation for this discrepancy is that growth of the Buneman-like and two-stream instabilities described above requires that the reflected ions form a distribution function with a positive gradient at some velocity (Laming 2000). This distribution forms when specularly reflected ions have a mostly monoenergetic, beamlike configuration. At the low Mach numbers in the solar wind $(\lesssim 10)$, where the shock structure is laminar, the reflected ions closely resemble a monoenergetic beam. However, at the higher Mach numbers, where the shock front is more turbulent and disordered, the reflected ions are likely to have a greater spread in energy and are probably less beamlike (Laming 2000). This would lead to suppression of Buneman-like instabilities. However, this line of reasoning is still speculative, and the real explanation for the lack of agreement between the observed $T_{e} / T_{p}$ and those predicted by models in this section remains to be explored. Cosmic-ray driven processes may ultimately provide a better explanation for electron heating at SNR shocks than those involving reflected suprathermal ions.

The cross shock potential arises from the charge separation produced by the different gyroradii for ions and electrons as they cross the shock transition. It accelerates electrons into the post shock layer, and can be a means of electron heating at subcritical shocks with an approximately laminar structure. At supercritical shocks, the time dependence and non-locality introduced reduces the degree of electron heating. However at sufficiently Alfvén Mach number (where the shock transition becomes thin (on the order of the electron convective gyroradius or inertial length), significant electron heating may again occur. In the absence of magnetic field amplification by cosmic rays, this might be expected to happen at SNR shocks. However it is much more likely in environments where the plasma beta is low, such as galaxy clusters. It may also occur in cases where a significant population of cosmic rays is unlikely due to the low age of the shock, as in gamma-ray bursts.

\section{Constraints From Solar Wind Studies}

From the beginning, a detailed study of the fastest collisionless shocks has been hampered by one inherent limitation: they occur in objects which are too remote for in situ study. Although some collisionless shocks in our solar system reach Alfvénic Mach numbers as high as 30 (such as those around Saturn; Achilleos et al. 2006; Masters et al. 2011), there are no physical phenomena in our solar system energetic enough to produce the type of shocks seen in SNRs (Mach numbers $\sim 100$ or more if no preshock magnetic field enhancement in the $\mathrm{CR}$ is assumed). In addition, the range of plasma betas attainable in the solar system is larger than the range of plasma betas attained in the interstellar medium.

Another fundamental difference between solar wind and SNR shocks is the fact that the former are short-lived phenomena confined to small spatial scales (millions of 
$\mathrm{km}$ ) in a curved (bow shock) geometry, whereas the latter are sustained for thousands of years, on spatial scales of parsecs, often well-described by a planar geometry. This results in an irreducible difference between the two types of shocks: particles crossing back and forth between upstream and downstream can remain in contact with SNR shocks for long periods of time, allowing accelerated CRs to acquire much more energy in SNR shocks than in solar wind shocks. This potentially allows the CRs to create shock precursors with properties needed to heat electrons and influence $T_{e} / T_{p}$.

The heating of electrons at the Earth's and other planetary bow shocks has been the subject of much theoretical and observational work. Typical features of the electron temperature change, $\Delta T_{e}$, observed at the bow shock as observed by Schwartz et al. (1988) include (a) an approximate relationship between heating and the incident solar wind energy $\Delta T_{e} \propto U^{2}$, where $U$ is the component of the solar wind's velocity incident upon the shock, and (b) a relationship between the change in temperature normalized by the incident energy and the fast magnetosonic Mach number, $\Delta T_{e} /\left(m_{p} U^{2} / 2\right) \propto M_{m s}^{-1}$. A similar approximate relationship holds between the normalized electron temperature change and the Alfvén Mach number $M_{A}$, especially for shocks with a low plasma $\beta$, which is the ratio between thermal and magnetic pressures. Recent work by Masters et al. (2011) shows that this relationship with $M_{A}$ holds well at Saturn's bow shock. This is particularly interesting as $M_{A}$ at Saturn is often much larger than at Earth.

In Figure 7 we plot the ratio of electron and ion temperatures downstream of the shock against the magnetosonic, and Alfvén Mach numbers, as well as the upstream flow velocity relative to the shock. The data in these figures are taken from bow shock crossings of the ISEE spacecraft, and is a subset of those listed in Schwartz et al. (1988), consisting of 61 crossings for which all the necessary data is available.

It is well known that quantities other than those displayed here may be more appropriate, specifically the change in electron temperature over the change in total temperature $\Delta T_{e} / \Delta\left(T_{e}+T_{i}\right)$ or even $\Delta T_{e} / \Delta T_{i}$ are better correlated with inverse Mach numbers than $T_{e} / T_{i}$ (Schwartz et al. 1988). Nevertheless, we use the latter quantity here as it enables a comparison with data from extra-solar system and outer planetary shocks at where less data are available. Furthermore, the approximate inverse dependence upon $M_{m s}, M_{A}$, and $V_{s}$ is still quite apparent in this data. It is interesting to note that the relationship with the Mach numbers is much more favourable than the dependence upon $V_{s}$, indicating that the Mach number is the more relevant quantity for organizing the relationship between $T_{e} / T_{p}$ and shock strength.

Many mechanisms may be involved in the heating of electrons in solar system shocks. Proposed mechanisms involve acceleration of electrons by a cross-shock potential (Goodrich and Scudder 1984; Scudder et al. 1986a 1986b), wave turbulence (Galeev 1976), microinstabilities (Wu et al. 1984), and electron trajectory scattering (Balikhin et al. 1993). The existence of a cross-shock potential may be deduced from the generalized Ohm's law in which a gradient in electron thermal pressure gives rise to an electric field. Examining the energetics of electrons crossing the shock may simplified by working in the de Hoffmann-Teller frame of reference (de Hoffmann and Teller 1950), defined as the frame in which the shock is at rest, and in which the magnetic field and plasma flow velocity are (anti-)parallel. In this case the the electric field is dominated by that generated by the electron pressure gradient, and the work done on electrons crossing the shock is determined by the cross-shock potential. Additional mechanisms are required to scatter electrons to pitch angles that are more perpendicular, and to flatten the distribution so that empty regions of phase space are filled. This results in a distribution 


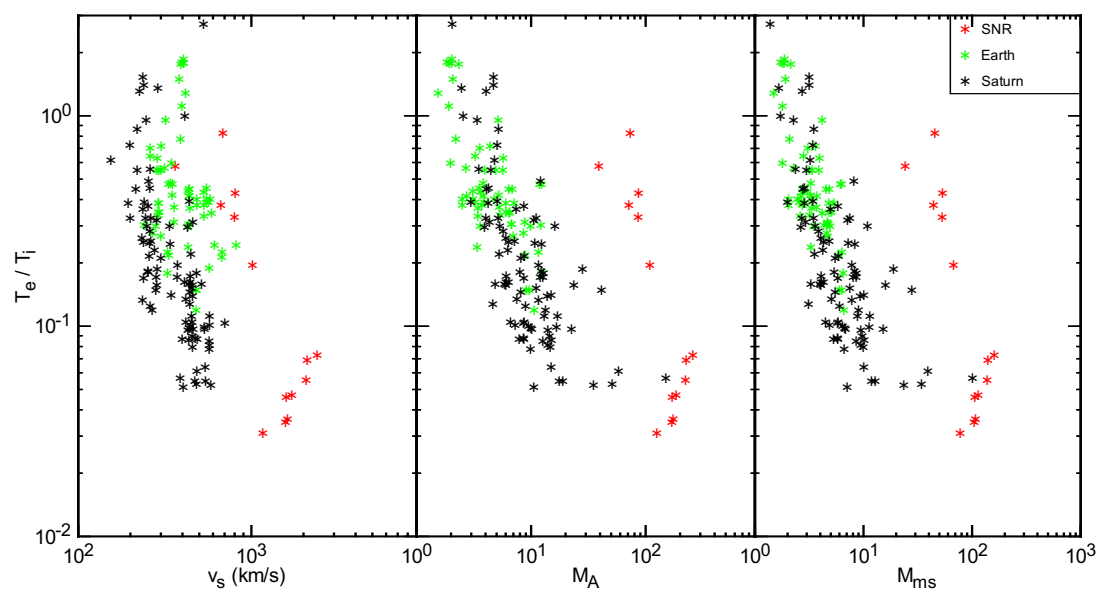

Fig. 7 Collected electron-ion equilibration data from both the solar wind bow shocks and supernova remnant shocks. $T_{e} / T_{p}$ is plotted versus shock speed (left), Alfvénic Mach number (center) and magnetosonic Mach number (right). Green symbols show data from crossings of Earth's bow shock (Schwartz et al. (1988)), while the black symbols show data from crossings of Saturn's bow shock (Masters et al. (2011)). Shock speeds for the Saturnian bow shock are based on a solar wind model and an assumed shock speed with respect to the spacecraft of $100 \mathrm{~km} \mathrm{~s}^{-1}$, and ion temperatures are based on electron distribution measurements and the application of the Rankine-Hugoniot conditions (see Masters et al. (2011) for a full discussion of shock parameter derivations at Saturn's bow shock). Red symbols show data acquired from Balmer-dominated SNR shocks (van Adelsberg et al. 2008), and assume $v_{A}=9 \mathrm{~km} \mathrm{~s}^{-1}, c_{s}=11$ $\mathrm{km} \mathrm{s}^{-1}$.

whose temperature is controlled to a large extent by the de Hoffmann-Teller frame cross-shock potential and downstream density. In addition to direct measurement of the electric fields within the shock, (Bale and Mozer 2007; Dimmock et al. 2011), comparison of upstream and downstream electron phase space distributions have shown that these are consistent with electron acceleration by a cross-shock potential in the de Hoffmann-Teller frame (Lefebvre et al. 2007).

\section{Connecting the Solar Wind Results to those in SNRs}

Figure 7 may indicate that similar mechanism(s) heat the electrons in solar wind and in SNR shocks. This is especially appealing when we remember that $M_{A}$ in SNRs may be overestimated due to preshock amplification of magnetic field by cosmic rays. In their study of the terrestrial bow shock and interplanetary shocks, Schwartz et al (1988) found that the electron-ion temperature equilibration organizes best by $T_{e} / T_{i} \propto 1 / M_{A}$. Given the difficulty in determining the Mach numbers of SNR shocks, the equilibration dependence on shock strength has been characterized via the shock speed instead, and 
found to obey $T_{e} / T_{i} \propto 1 / V_{s}^{2}$ ( $V_{s}$ is much more accurately known than the Mach numbers). Subsection 4.1 outlined a model for SNR electron heating, where the cosmic ray diffusion coefficient $\kappa_{C R}$ is assumed independent of $V_{s}$. From quasi-linear theory (Blandford and Eichler 1987),

$$
\kappa_{C R}=\frac{p^{2} c^{2} v}{3 \pi e^{2} U}
$$

where $U$ is the energy density of turbulence $\left(\equiv\left\langle\Delta B^{2}\right\rangle / 8 \pi\right)$ and $v$ is the cosmic ray velocity. For resonant amplification, we evaluate $U$ at $k_{\|}=\Omega / v_{\|}$, where $\Omega$ is the gyroradius and $v_{\|}$the parallel component of the cosmic ray velocity. For relativistic cosmic rays, where $v=c$, this results in $\kappa_{C R} \propto p^{2} / U$, assumed constant with $V_{s}$. However, for nonrelativistic suprathermal particles, $v$ will most likely be proportional to the shock velocity $V_{s}$, which with the same assumptions leads to $T_{e} / T_{i} \propto 1 / V_{s}$ for solar wind shocks (as opposed to $1 / V_{s}^{2}$ in SNRs). This argument is admittedly loose, and should not be be viewed as much more than a hypothesis to motivate further work.

In our arguments above we have made considerable assumptions about $\kappa_{C R}$. The most obvious one is that $\kappa_{C R}$ as written above applies to parallel shocks, whereas we are most likely dealing with quasi-perpendicular cases. This may reduce the difference anticipated between solar wind and SMR shocks, depending on the turbulence spectrum (summarized in Appendix A of Rakowski et al. 2008).

The degree of cosmic ray magnetic field amplification at SNR shocks required to bring SNR data points in Figure 7 into alignment with solar wind data points is approximately an order of magnitude or less at the highest velocities considered. Given the degree of magnetic field amplification expected from cosmic ray acceleration, this appears to be highly plausible. In the case of saturated nonresonant instability (Bell 2004,2005 ) by resonant scattering (Luo \& Melrose 2009), $\langle\Delta B\rangle^{2} / B^{2} \sim 10-100$ is expected. In the case of nonresonant saturation, higher values, but strongly dependent on $k$, are predicted. Saturation by electron heating (i.e. the $M_{A}$ where growth of lower hybrid waves becomes greater than the growth rate of magnetic field) leads to similar magnetic field enhancement, with $\Delta B^{2} / B \sim 200$ (Rakowski et al. 2008). Such magnetic field amplification is less likely at solar wind shocks. The suprathermal particle densities are lower in solar wind shocks, and the ambient magnetic fields are higher, much closer to where the nonresonant instability would saturate (if not already beyond it).

\section{Observational Constraints from Galaxy Cluster Shocks}

Collisionless shocks occur over a vast range of length scales, with those in galaxy clusters being among the largest. While the shock speeds in the galaxy cluster shocks are similar to those in supernova remnants (up to $4000 \mathrm{~km} \mathrm{~s}^{-1}$; Markevitch 2005; Markevitch \& Vikhlinin 2007; Russell et al. 2012), they occur in environments that are substantially different from both the solar wind and the ISM. These differences can be encapsulated via the plasma beta, defined as the ratio of the thermal pressure to the magnetic pressure of a plasma $\left(\beta \equiv n k T /\left(B^{2} / 8 \pi\right)\right)$. Utilizing solar wind parameters listed by Bruno \& Carbone (2005), this ratio ranges from around unity at 1 AU under fast solar wind conditions (wind velocity $\sim 900 \mathrm{~km} \mathrm{~s}^{-1}$ ) to around 20 for the quiescent wind (wind velocity $\sim 300 \mathrm{~km} \mathrm{~s}^{-1}$ ). The plasma $\beta$ of the ISM is close to that of the fast solar wind, $\beta_{I S M} \sim 1-4$ (assuming a $\mathrm{B}=3 \mu \mathrm{G}, \mathrm{n}=1 \mathrm{~cm}^{-3}$ and $\mathrm{T}=10^{4} \mathrm{~K}$ ). On 
the other hand, the electron temperature of the intracluster medium (ICM) ranges from $10^{7} \mathrm{~K}-10^{8} \mathrm{~K}(1-10 \mathrm{keV})$, with number densities steeply declining from $\sim 10^{2}$ $\mathrm{cm}^{3}$ near the cluster centers to $\sim 10^{4} \mathrm{~cm}^{3}$ at the outer edges. The corresponding sound speed is close to $1000 \mathrm{~km} \mathrm{~s}^{-1}$, nearly two orders of magnitude higher than in the general ISM. The magnetic fields measured in galaxy clusters are actually close that of the ISM, typically on the order of a microGauss (Carilli \& Taylor 2002). Therefore, $v_{A} \sim 50 \mathrm{~km} \mathrm{~s}^{-1}$ in galaxy clusters, so that $\beta_{e} \gg 1$ and the magnetic field pressure has negligible contribution to the dynamics of shocks in galaxy clusters. This puts galaxy cluster shocks in a different region of parameter space than solar wind and SNR shocks.

Clusters such as 1E0657-56 (the 'Bullet Cluster') and A520 show strongly enhanced X-ray emission from collisionless shocks, formed during major mergers when gas from one cluster plunges through gas from the other (Markevitch et al. 2005; Markevitch \& Vikhlinin 2007; Russell et al. 2012). Shocks moving mostly along the plane of the sky have a favorable viewing geometry and appear as giant curved structures hundreds of kiloparsecs in length. The large clumps of infalling gas drive bow shocks into the cluster gas, which has already been heated to at least $1 \mathrm{keV}$, and produces thermal Bremsstrahlung emission peaking close to that energy. This is another major difference between collisionless shocks in the ISM and those in the ICM. While the Alfvénic and magnetosonic Mach numbers of SNR shocks are very difficult to determine due to the lack of available observational constraints on magnetohydrodynamic quantities upstream (such as $T, B$ and $n$ ), those in galaxy clusters can readily be measured by spectral analysis of X-ray emission upstream. Comparison of this emission to that of the enhanced postshock region gives the density contrast between the downstream and upstream gas (i.e., $n_{2} / n_{1}$ ). This density contrast yields the sonic Mach number, $M$, via solving the Rankine-Hugoniot jump conditions (Russell et al. 2012):

$$
M=\left(\frac{2 n_{2} / n_{1}}{\gamma+1-\frac{n_{2}}{n_{1}}(\gamma-1)}\right)^{1 / 2}
$$

where $\gamma$ is the ratio of specific heats of the cluster gas. Measurement of these jumps from X-ray observations have yielded $M \sim 1.5-3$ for the Bullet Cluster (Markevitch et al. 2005), Abell 520 and Abell 2146 (Russell et al. 2012). Using these estimated Mach numbers, the shock velocity itself can be calculated via $V_{s}=M c_{s}$, where $c_{s}$ is the upstream sound speed as inferred from the X-ray spectra. This yields shock speeds ranging between $2500 \mathrm{~km} \mathrm{~s}^{-1}$ and $4000 \mathrm{~km} \mathrm{~s}^{-1}$, similar to the fastest known Balmerdominated shocks. However, fits to the X-ray spectra behind these shocks show prompt electron-ion equilibration at the shock front, consistent with $T_{e} / T_{p}=1$ (Markevitch et al. 2005; Markevitch \& Vikhlinin 2007), despite the extremely high shock speeds involved. This result can only be reconciled with equilibration measurements from the solar wind and SNRs if the equilibration depends on the Mach number, rather than $V_{s}$. Furthermore, given the low Mach numbers found in the galaxy clusters, it is plausible that the shock transitions in these cases are laminar (as opposed to turbulent like the SNR and fastest solar wind shocks), with electron heating occurring efficiently at the shock front via the same type of cross-shock potential as seen in the slowest solar wind and slowest $\mathrm{SNR}\left(V_{s} \leq 400 \mathrm{~km} \mathrm{~s}^{-1}\right)$ shocks. This is of course a speculation; further insight into collisionless cluster shocks may be obtained via numerical simulations for the appropriate conditions. 


\section{Summary and Future Work}

There have been exciting advances in the study of electron-ion temperature equilibration in collisionless shocks during the past few years. Perhaps most notable has been the growing realization that temperature equilibration and cosmic ray acceleration may be intertwined processes. Optical studies of collisionless shocks in partially neutral gas (Balmer-dominated shocks) have shown that the electron-ion temperature equilibration is a declining function of shock speed, well characterized as $T_{e} / T_{p} \propto V_{s}^{-2}$. This relationship most likely arises due to electron heating ahead of the shock that is nearly independent of shock speed above $400 \mathrm{~km} \mathrm{~s}^{-1}$. Cosmic ray precursors, with moderately amplified preshock magnetic field and density, are the most logical sites for electron heating in SNR collisionless shocks. The transition to fully equilibrated SNR shocks at speeds below $400 \mathrm{~km} \mathrm{~s}^{-1}$ may be due to a less turbulent and more laminar structure at low shock speeds and Mach numbers. This allows the electrons to experience a more uniform cross-shock potential, and hence higher energization compared to higher shock speeds and Mach numbers, where the shock jump is more turbulent and disordered. The magnetosonic Mach numbers of SNR shocks may match those in solar wind shocks if there is a approximately an order of magnitude increase in the Alfvén speed of the preshock gas in SNRs compared to the average ISM value. This is possible if there is a moderately amplified preshock magnetic field $(\sim 10)$, and is readily provided by compression and heating in a cosmic ray precursor. In solar wind shocks, the precursor is due to suprathermal, non-relativistic ions, resulting in a $T_{e} / T_{p} \propto 1 / V_{s}$ seen in the solar wind.

While Balmer-dominated shocks have allowed us to elucidate some of the physics of electron-ion temperature equilibration, ion-neutral damping limits most of those observed to cases where the shock structure has not become strongly modified by cosmic ray acceleration. Given this limitation, electron-ion equilibration studies of fast, collisionless shocks in fully pre-ionized gas would be highly desirable. Such a sample would allow the equilibration to be studied over a range of speeds where shocks are increasingly affected by feedback from the accelerated cosmic rays. In such circumstances it is unclear what would happen to the $T_{e} / T_{p}$ versus $V_{s}$ relation. If, as predicted by Amato \& Blasi (2009), Bell's non-resonant cosmic ray instability takes over from the resonant instability at the highest shock speeds, then additional electron heating may occur in the fastest shocks $\left(V_{s} \gtrsim 5000 \mathrm{~km} \mathrm{~s}^{-1}\right)$, resulting in substantial deviation from the $T_{e} / T_{p} \propto V_{s}^{-2}$ relation. Such deviations may already have been seen in the fastest $\left(V_{s} \gtrsim 2000 \mathrm{~km} \mathrm{~s}^{-1}\right.$ ) shocks, where there is evidence that $T_{e} / T_{p}$ does not settle down to $m_{e} / m_{p}$, but rather $\sim 0.03$. Other deviations may have been detected in SNR 0509-67.5, where $T_{e} / T_{p}$ for a $5000 \mathrm{~km} \mathrm{~s}^{-1}$ shock has been estimated to be $\sim 0.2$, substantially higher than predicted by the inverse squared relation. However, the study of such shocks would be challenging. Without an $\mathrm{H} \alpha$ broad component to constrain the range of $V_{s}$, shock speeds would have to be determined by other means, such as proper motion studies. That would require X-ray and or UV imagery of SNRs with well-constrained distances (such as those in the LMC or SMC), over multiple epochs. It would also require spectroscopy of the forward shocks in these SNRs, in order to constrain both the electron temperature (via X-ray continuum fits and and UV emission line ratios) and the ion temperature (e.g., via He II, C IV, N V and O VI resonance lines).

An important test of our ideas concerning electron heating by cosmic ray generated waves in a shock precursor would be to measure electron temperatures at SNR shocks 
exhibiting strong cosmic ray modification and substantial magnetic field amplification $(\Delta B / B \gtrsim 100)$. Several SNRs show X-ray filaments produced by synchrotron radiation from cosmic ray electrons, and are generally distinct from those shocks with strong Balmer emission. The absence of neutral material ahead of these shock means that optical and UV emission is weak, and electron temperatures will have to be measured from X-ray spectra. Difficulties arise in distinguishing thermal electron bremsstrahlung from cosmic ray electron synchrotron emission, requiring data of high signal to noise. Further complications in some SNRS (e.g. Cas A) stem from scattering of bright Xray emission from the ejecta such that it coincides spatially with emission from the forward shock. Such scattering may either be local, due to SNR dust, or instrumental, due to telescope imperfections. This leaves SN 1006 as the best likely target for such an observation, since due to its evolutionary state, only the outer layers of ejecta have encountered the reverse shock.

The development of missions like Solar Orbiter and Solar Probe Plus will allow in situ measurements of shocks in the solar wind, most likely associated with coronal mass ejections, much closer to the Sun. These will probe a different parameter regime, where the magnetic field pressure dominates over the gas pressure (low $\beta$, similar to ISM shocks). As such, measurements here might yield insights into the properties of similar plasma in the precursors of SNR shocks where the magnetic field has been amplified by cosmic rays.

Acknowledgements P. G. acknowledges support by HST grant HST-GO-11184.07-A to Towson University. JML acknowledges support by grant NNH10A009I from the NASA Astrophysics Data Analysis Program, and by basic research funds of the Office of Naval Research.

\section{References}

A. A. Abdo, M. Ackermann, M. Ajello et al.. Observations of the Young Supernova Remnant RX J1713.7-3946 with the Fermi Large Area Telescope. Astrophysical Journal, 734:28, June 2011. doi: 10.1088/0004-637X/734/1/28.

F. Acero, F. Aharonian, A. G. Akhperjanian et al.. First detection of VHE $\gamma$-rays from SN 1006 by HESS. Astronomy and Astrophysics, 516:A62, June 2010. doi: 10.1051/0004-6361/ 200913916

N. Achilleos, C. Bertucci, C. T. Russell et al.. Orientation, location and velocity of Saturn's bow shock: initial results from the Cassini spacecraft. Journal of Geophysical Research, 111:A03201. doi: doi:10.1029/2005JA011297.

F. Aharonian et al.. On the origin of TeV Radiation of SN 1006. Astronomy and Astrophysics, 351:330, Nov 1999. doi: .

F. Aharonian, A. G. Akhperjanian, A. R. Bazer-Bachi et al.. A detailed spectral and morphological study of the gamma-ray supernova remnant RX J1713.7-3946 with HESS. Astronomy and Astrophysics, 449:223-242, April 2006. doi: 10.1051/0004-6361:20054279.

T. Amano and M. Hoshino. A Critical Mach Number for Electron Injection in Collisionless Shocks. Physical Review Letters, 104(20):181102, May 2010. doi: 10.1103/PhysRevLett. 104.181102.

E. Amato and P. Blasi. A kinetic approach to cosmic-ray-induced streaming instability at supernova shocks. Monthly Notices of the Royal Astronomical Society, 3921591-1600, February 2009. doi: 10.1111/j.1365-2966.2008.14200.x.

S. D. Bale and F. S. Mozer. Measurement of Large Parallel and Perpendicular Electric Fields on Electron Spatial Scales in the Terrestrial Bow Shock. Physical Review Letters, 98(20): 205001, May 2007. doi: 10.1103/PhysRevLett.98.205001.

M. Balikhin, M. Gedalin, and A. Petrukovich. New mechanism for electron heating in shocks. Physical Review Letters, 70:1259-1262, March 1993. doi: 10.1103/PhysRevLett.70.1259. 
J. Ballet. X-ray synchrotron emission from supernova remnants. Advances in Space Research, 37:1902-1908, 2006. doi: 10.1016/j.asr.2005.03.047.

A. Bamba, R. Yamazaki, T. Yoshida, T. Terasawa, and K. Koyama. A Spatial and Spectral Study of Nonthermal Filaments in Historical Supernova Remnants: Observational Results with Chandra. Astrophysical Journal, 621:793-802, March 2005. doi: 10.1086/427620.

A. R. Bell and S. G. Lucek. Cosmic ray acceleration to very high energy through the nonlinear amplification by cosmic rays of the seed magnetic field. Monthly Notices of the Royal Astronomical Society, 321:433-438, March 2001. doi: 10.1046/j.1365-8711.2001.04063.x.

A. R. Bell. Turbulent amplification of magnetic field and diffusive shock acceleration of cosmic rays. Monthly Notices of the Royal Astronomical Society, 353:550-558, March 2005. doi: 10.1111/j.1365-2966.2004.08097.x.

A. R. Bell. The interaction of cosmic rays and magnetized plasma. Monthly Notices of the Royal Astronomical Society, 358:181-187, September 2004. doi: 10.1111/j.1365-2966.2005. 08774.x.

R. Blandford and D. Eichler. Particle acceleration at astrophysical shocks: A theory of cosmic ray origin. Physics Reports, 154:1-75, October 1987. doi: 10.1016/0370-1573(87)90134-7.

P. Blasi, G. Morlino, R. Bandiera, E. Amato, and D. Caprioli. Collisionless Shocks in a Partially Ionized Medium. I. Neutral Return Flux and its Effects on Acceleration of Test Particles. Astrophysical Journal, 755:121, August 2012. doi: 10.1088/0004-637X/755/2/121.

E. G. Berezhko, L. T. Ksenofontov, and H. J. Völk. Confirmation of strong magnetic field amplification and nuclear cosmic ray acceleration in SN 1006. Astronomy and Astrophysics, 412:L11-L14, December 2003. doi: 10.1051/0004-6361:20031667.

R. Bruno and V. Carbone. The Solar Wind as a Turbulence Laboratory. Living Reviews in Solar Physics, 2:4, September 2005. doi: .

P. J. Cargill and K. Papadopoulos. A mechanism for strong shock electron heating in supernova remnants. Astrophysical Journal, 329:L29-L32, June 1988. doi: 10.1086/185170.

C. L. Carilli and G. B. Taylor(2002). Cluster Magnetic Fields. Annual Review of Astronomy and Astrophysics, 40:319-348, 2002. doi: 10.1146/annurev.astro.40.060401.093852.

R. A. Chevalier and J. C. Raymond. Optical emission from a fast shock wave - The remnants of Tycho's supernova and SN 1006. Astrophysical Journal, 225:L27-L30, October 1978. doi: $10.1086 / 182785$.

R. A. Chevalier, R. P. Kirshner, and J. C. Raymond. The optical emission from a fast shock wave with application to supernova remnants. Astrophysical Journal, 235:186-195, January 1980. doi: 10.1086/157623.

D. P. Cox and J. C. Raymond. Preionization-dependent families of radiative shock waves. Astrophysical Journal, 298:651-659, November 1985. doi: 10.1086/163649.

F. de Hoffmann and E. Teller. Magneto-Hydrodynamic Shocks. Physical Review, 80:692-703, November 1950. doi: 10.1103/PhysRev.80.692.

A. Decourchelle and D. Ellison. Thermal X-Ray Emission and Cosmic-Ray Production in Young Supernova Remnants. Astrophysical Journal Letters, 543:L57-L60, November 2000. doi: $10.1086 / 318167$.

M. E. Dieckmann, A. Bret, G. Sari, E. Perez Alvaro, I. Kourakis, and M. Borghesi. Particle simulation study of electron heating by counter-streaming ion beams ahead of supernova remnant shocks. Plasma Physics and Controlled Fusion, 54:085015, August 20122. doi: 10.1088/0741-3335/54/8/085015.

A. P. Dimmock, M. A. Balikhin, and Y. Hobara. Comparison of three methods for the estimation of cross-shock electric potential using Cluster data. Annales Geophysicae, 29: 815-822, May 2011. doi: 10.5194/angeo-29-815-2011.

B. T. Draine and C. F. McKee. Theory of Interstellar Shocks. Annual review of astronomy and astrophysics, 31:3733-432, 1993. doi: 10.1146/annurev.aa.31.090193.002105.

L. O'C. Drury, P. Duffy, and J. G. Kirk. Limits on diffusive shock acceleration in dense and incompletely ionised media.. Astronomy and Astrophysics, 309:1002-1010, May 1996. doi:

J. P. Edmiston, and C. F. Kennel. A parametric survey of the first critical Mach number for a fast MHD shock. Journal of Plasma Physics, 32:429-441, December 1984. doi: $10.1017 /$ S002237780000218X.

D. C. Ellison, D. J. Patnaude, P. Slane, P. Blasi and S. Gabici. Particle Acceleration in Supernova Remnants and the Production of Thermal and Nonthermal Radiation. Astrophysical Journal, 661:879-891, June 2007. doi: 10.1086/517518.

K. A. Eriksen, J. P. Hughes, C. Badenes, R. Fesen, P. Ghavamian, D. Moffett, P. P. Plucinsky, C. E. Rakowski, E. M. Reynoso, and P. Slane. Evidence for Particle Acceleration to the 
Knee of the Cosmic Ray Spectrum in Tycho's Supernova Remnant. Astrophysical Journal, 728:L28, February 2011. doi: 10.1088/2041-8205/728/2/L28.

K. France, R. McCray, S. V. Penton, R. P. Kirshner, P. Challis, J. M. Laming, P. Bouchet, R. Chevalier, P. M. Garnavich, C. Fransson, K. Heng, J. Larsson, S. Lawrence, P. Lundqvist, N. Panagia, C. S. J. Pun, N. Smith, J. Sollerman, G. Sonneborn, B. Sugerman, and J. C. Wheeler HST-COS Observations of Hydrogen, Helium, Carbon, and Nitrogen Emission from the SN 1987A Reverse Shock. Astrophysical Journal, 743:186, December 2011. doi: 10.1088/0004-637X/743/2/186.

A. A. Galeev. Collisionless shocks. In D. J. Williams, editor, Physics of Solar Planetary Environments, pages 464-490, 1976.

M. Gedalin, M. A. Balikhin, and D. Eichler. Efficient electron heating in relativistic shocks and gamma-ray-burst afterglow. Physical Review E, 77:026403, February 2008. doi: 10. 1103/PhysRevE.77.026403.

P. Ghavamian. Optical Spectroscopy and Numerical Models of Nonradiative Shocks in Supernova Remnants. PhD Thesis, Rice University December 1999.

P. Ghavamian, J. C. Raymond, P. Hartigan, and W. P. Blair. Evidence for Shock Precursors in Tycho's Supernova Remnant. Astrophysical Journal, 535:266-2274, May 2000. doi: $10.1086 / 308811$.

P. Ghavamian, J. C. Raymond, R. C. Smith, and P. Hartigan Balmer-dominated Spectra of Nonradiative Shocks in the Cygnus Loop, RCW 86, and Tycho Supernova Remnants. Astrophysical Journal, 547:995-1009, February 2001. doi: 10.1086/318408.

P. Ghavamian, P. F. Winkler, J. C. Raymond, and K. S. Long The Optical Spectrum of the SN 1006 Supernova Remnant Revisited. Astrophysical Journal, 572:888-896, June 2002. doi: $10.1086 / 340437$.

P. Ghavamian, C. E. Rakowski, J. P. Hughes, and T. B. Williams The Physics of Supernova Blast Waves. I. Kinematics of DEM L71 in the Large Magellanic Cloud. Astrophysical Journal, 590:833-845, June 2003. doi: 10.1086/375161.

P. Ghavamian, J. M. Laming, and C. E. Rakowski. A Physical Relationship between ElectronProton Temperature Equilibration and Mach Number in Fast Collisionless Shocks. Astrophysical Journal, 654:L69-L72, January 2007. doi: 10.1086/510740.

C. C. Goodrich and J. D. Scudder. The adiabatic energy change of plasma electrons and the frame dependence of the cross-shock potential at collisionless magnetosonic shock waves. Journal of Geophysical Research, 89:6654-6662, August 1984. doi: 10.1029/ JA089iA08p06654.

E. Gosset, M. De Becker, Y. Nazé, S. Carpano, G. Rauw, I. I. Antokhin, J.-M. Vreuz, and A. M. T. Pollock. XMM-Newton observation of the enigmatic object WR 46. Astronomy and Astrophysics, 527:A66, March 2011. doi: 10.1051/0004-6361/200912510.

E. W. Greenstadt and M. M. Mellott. Plasma wave evidence for reflected ions in front of subcritical shocks - ISEE 1 and 2 observations. Journal of Geophysical Research , 92: 4730-4734, May 1987. doi: 10.1029/JA092iA05p04730.

E. A. Helder, J. Vink, C. GH. Bassa, A. Bamba, J. A. M. Bleeker, S. Funk, P. Ghavamian, K. J. van der Heyden, F. Verbunt, and R. Yamazaki. Measuring the Cosmic-Ray Acceleration Efficiency of a Supernova Remnant. Science, 325:719, August 2009. doi: 10.1126/science.1173383.

E. A. Helder, D. Kosenko, and J. Vink. Cosmic Ray Acceleration Efficiency versus Temperature Equilibration: The Case of SNR 0509-67.5. Astrophysical Journal Letters, 719:L140, August 2010 doi: 10.1088/2041-8205/719/2/L140.

E. A. Helder, J. Vink and C. G. Bassa.. Temperature Equilibration Behind the Shock Front: An Optical and X-ray Study of RCW 86. Astrophysical Journal, 737:85, August 2011 doi: 10.1088/0004-637X/737/2/85.

K. Heng and R. McCray. Balmer Dominated Shocks Revisited. Astrophysical Journal, 654: 923-937, January 2007. doi: 10.1086/509601.

K. Heng, M. van Adellsberg, R. McCray, and J. C. Raymond. The Transition Zone in Balmer Dominated Shocks. Astrophysical Journal, 668:275-284, October 2007. doi: 10.1086/ 521298.

J. J. Hester, J. C. Raymond, and W. P. Blair. The Balmer-dominated northeast limb of the Cygnus loop supernova remnant. Astrophysical Journal, 420:721-745, January 1994. doi: $10.1086 / 173598$

H. Itoh. Two-fluid blast-wave model for supernova remnants.. Publications of the Astronomical Society of Japan, 30:489-498, 1978. doi: . 
F. C. Jones and D. C. Ellison. The plasma physics of shock acceleration. Space Science Reviews, 58:259-346, December 1991. doi: 10.1007/BF01206003.

C. F. F. Karney. Stochastic ion heating by a lower hybrid wave. Physics of Fluids, 21: 1584-1599, September 1978. doi: 10.1063/1.862406.

S. Katsuda, R. Petre, K. Mori, S. P. Reynolds, K. S. Long, P. F. Winkler, and H. Tsunemi. Steady X-ray Synchrotron Emission in the Northeastern Limb of SN 1006. Astrophysical Journal, 723:383-392, November 2010. doi: 10.1088/0004-637X/723/1/383.

S. Katsuda, R. Petre, J. P. Hughes, U. Hwang, H. Yamagauchi, A. Hayato, K. Mori, and H. Tsunemi. X-ray Measured Dynamics of Tycho's Supernova Remnant. Astrophysical Journal, 709:1387-1395, February 2010. doi: 10.1088/0004-637X/709/2/1387.

S. Katsuda, K. S. Long, R. Petre, S. P. Reynolds, B. J. Williams, and P. F. Winkler. X-ray Proper Motions and Shock Speeds along the Northwest Rim of SN 1006. arXiv:1211.6443 2012. doi: .

C. F. Kennel, J. P. Edmiston and T. Hada. A quarter century of collisionless shock research. Washington DC American Geophysical Union Monograph Series, 34:1-36 doi: .

R. Kirshner, P. F. Winkler, and R. A. Chevalier. High-velocity emission in young supernova remnants: SN 1006 and SN 1572. Astrophysical Journal, 315:L135-L139, April 1987. doi: $10.1086 / 184875$

K. E. Korreck, J. C. Raymond, T. H. Zurbuchen and P. Ghavamian. Far Ultraviolet Spectroscopic Explorer Observation of the Nonradiative Collisionless Shock in the Remnant of SN 1006. Astrophysical Journal, 615:280-285, November 2004. doi: 10.1086/424481.

K. Koyama, R. Petre, E. V. Gotthelf, U. Hwang, M. Matsuura, M. Ozaki, and S. S. Holt. Evidence for shock acceleration of high-energy electrons in the supernova remnant SN1006. Nature, 378:255-258, November 1995. doi: 10.1038/378255a0.

K. Koyama, K. Kinugasa, K. Matsuzaki, M. Nishiuchi, M. Sugizaki, K. Torii, S. Yamauchi, and B. Aschenbach. Discovery of Non-Thermal X-Rays from the Northwest Shell of the New SNR RX J1713.7-3946: The Second SN 1006?. Publications of the Astronomical Society of Japan, 49:L7-L11, June 1997. doi: .

V. V. Krasnoselskikh, B. Lembège, P. Savoini and V. V Lobzin Nonstationarity of strong collisionless quasiperpendicular shocks: Theory and full particle numerical simulations. Physics of Plasmas, 9:1192-12090, April 2002. doi: 10.1063/1.1457465.

R. M. Kulsrud and C. J. Cesarsky. The Effectiveness of Instabilities for the Confinement of High Energy Cosmic Rays in the Galactic Disk. Astrophysical Letters, 8:189, March 1971. doi: .

J. M. Laming, J. C. Raymond, B. M. McLaughlin and W. P. Blair. Electron-Ion Equilibration in Nonradiative Shocks Associated with SN 1006. Astrophysical Journal, 472:267-274, November 1996. doi: 10.1086/178061.

J. M. Laming. Electron Heating at SNR Collisionless Shocks. Astrophysical Journal Supplement Series, 127:409-413, April 2000. doi: 10.1086/313325.

J. M. Laming. Accelerated Electrons in Cassiopeia A: An Explanation for the Hard X-Ray Tail. Astrophysical Journal, 546:1149-1158, January 2001. doi: 10.1086/318317.

J. J. Lee, B.-C. Koo, J. C. Raymond, P. Ghavamian, T.-S. Pyo, A. Tajitsu and M. Hayashi. Subaru HDS Observations of a Balmer-dominated Shock in Tycho's Supernova Remnant. Astrophysical Journal Letters, 659:L133-L136, April 2007. doi: 10.1086/517520.

J. J. Lee, J. C. Raymond, S. Park, W. P. Blair, P. Ghavamian, P. F. Winkler, K. Korreck. Resolved Shock Structure of the Balmer-dominated Filaments in Tycho's Supernova Remnant: Cosmic-ray Precursor?. Astrophysical Journal Letters, 715:L146-L149, June 2010. doi: 10.1088/2041-8205/715/2/L146.

B. Lefebvre, S. J. Schwartz, A. F. Fazakerley, and P. Décréau. Electron dynamics and crossshock potential at the quasi-perpendicular Earth's bow shock. Journal of Geophysical Research (Space Physics), 112:A09212, September 2007. doi: 10.1029/2007JA012277.

Q. Luo and D. Melrose. Saturated magnetic field amplification at supernova shocks. Monthly Notices of the Royal Astronomical Society, 397:1402-1409, August 2009. doi: 10.1111/j. 1365-2966.2009.14872.x.

M. Markevitch, F. Govoni, G. Brunetti, and D. Jerius. Bow Shock and Radio Halo in the Merging Cluster A520. Astrophysical Journal, 627:733-738, July 2005. doi: 10.1086/ 430695.

M. Markevitch and A. Vikhlinin. Shocks and cold fronts in galaxy clusters. Physics Reports, 443:1-53, May 2007. doi: 10.1016/j.physrep.2007.01.001. 
A. Masters, S. J. Schwartz, E. M. Henley, M. F. Thomsen, B. Zieger, A. J. Coates, N. Achilleos, J. Mitchell, K. C. Hansen, and M. K. Dougherty. Electron heating at Saturn's bow shock. Journal of Geophysical Research (Space Physics), 116:A10107, October 2011. doi: 10. 1029/2011JA016941.

S. Matsukiyo. Mach number dependence of electron heating in high Mach number quasiperpendicular shocks. Physics of Plasmas, 17:042901, April 2010. doi: 10.1063/1.3372137.

K. G. McClements, R. O. Dendy, R. Bingham, J. G. Kirk, and L. O'C. Drury. Acceleration of cosmic ray electrons by ion-excited waves at quasi-perpendicular shocks. Monthly Notices of the Royal Astronomical Society, 291:241-249, October 1997. doi: .

G. Morlino, E. Amato, P. Blasi, and D. Caprioli. Spatial structure of X-ray filaments in SN 1006. Monthly Notices of the Royal Astronomical Society, 405:L21-L25, June 2010. doi: 10.1111/j.1745-3933.2010.00851.x.

G. Morlino, R. Bandiera, P. Blasi, and E. Amato. Collisionless Shocks in a Partially Ionized Medium. II. Balmer Emission. Astrophysical Journal, 760:137, December 2012. doi: 10. 1088/0004-637X/760/2/137.

G. Morlino, P. Blasi, R. Bandiera, E. Amato, and D. Caprioli. Collisionless shocks in a partially ionized medium: III. Efficient cosmic ray acceleration. arXiv1211.6148, November 2012. doi: .

S. Orlando, F. Bocchino, F. Reale, F. Peres, and O. Petruk. On the origin of asymmetries in bilateral supernova remnants. Astronomy and Astrophysics, 470:927-939, August 2007. doi: 10.1051/0004-6361:20066045.

K. Papadopoulos. Comments on high Mach number magnetosonic shocks. ESASP, 161:409, November 1981. doi: .

K. Papadopoulos. Electron heating in superhigh Mach number shocks. Astrophysics and Space Science, 144:535-547, May 1988. doi: 10.1007/BF00793203.

O. Petruk, F. Bocchino, G. Castelletti, G. Dubner, D. Lakubovskyi, M. Kirsch, M. Miceli and I. Telezhinsky. X-ray emission of the shock of SN1006. Constraints on electron kinetics. Proc. "The X-ray Universe 2008", Granada, Spain, 109, July 2008. doi: .

C. E. Rakowski, P. Ghavamian and J. P. Hughes. The Physics of Supernova Remnant Blast Waves. II. Electron-Ion Equilibration in DEM L71 in the Large Magellanic Cloud. Astrophysical Journal, 590:846-857, June 2003 doi: 10.1086/375162

C. E. Rakowski, J. M. Laming, and P. Ghavamian. The Heating of Thermal Electron in Fast Collisionless Shocks: The Integral Role of Cosmic Rays. Astrophysical Journal, 684: 348-357, September 2008. doi: 10.1086/590245.

C. E. Rakowski, P. Ghavamian, and J. M. Laming. The $\mathrm{H} \alpha$ Diagnostic of Electron Heating: The Case of DEM L71. Astrophysical Journal, 696:2195-2205, May 2009. doi: 10.1088/ 0004-637X/696/2/2195.

J. C. Raymond, W. P. Blair, and K. S. Long. Detection of Ultraviolet Emission Lines in SN 1006 with the Hopkins Ultraviolet Telescope. Astrophysical Journal, 454:L31-L34, November 1995. doi: 10.1086/309772.

J. C. Raymond, J. Vink, E. A. Helder, and A. de Laat Effects of Neutral Hydrogen on Cosmicray Precursors in Supernova Remnant Shock Waves. Astrophysical Journal, 731:L14, April 2011. doi: 10.1088/2041-8205/731/1/L14.

B. Reville, J. G. Kirk, P. Duffy, and S. O'Sullivan. A cosmic ray current-driven instability in partially ionised media. Astronomy and Astrophysics, 475:435-439, November 2007. doi: 10.1051/0004-6361:20078336.

M. A. Riquelme and A. Spitkovsky. Electron Injection by Whistler Waves in Non-relativistic Shocks. Astrophysical Journal, 733:63, May 2011. doi: 10.1088/0004-637X/733/1/63.

H. R. Russell, B. R. McNamara, J. S. Sanders, A. C. Fabian, P. E. J. Nulsen, R. E. A. Canning, S. A. Baum, M. Donahue, A. Edge, L. J. King and C. P. O'Dea. Shock Fronts, Electron-Ion Equilibration and ICM Transport Processes in the Merging Cluster Abell 2146. Monthly Notices of the Royal Astronomical Society, 423:236-255, June 2012. doi: 10.1111/j.1365-2966.2012.20808.x.

K. M. Schure, A. R. Bell, L. O'C. Drury, and A. M. Bykov. Diffusive Shock Acceleration and Magnetic Field Amplification. Space Science Reviews, 173:491-519, November 2012. doi: 10.1007/s11214-012-9871-7.

S. J. Schwartz, M. F. Thomsen, S. J. Bame, and J. Stansberry. Electron heating and the potential jump across fast mode shocks. Journal of Geophysical Research, 93:12923-12931, November 1988. doi: 10.1029/JA093iA11p12923. 
S. J. Schwartz, E. G. Zweibel, and M. Goldman. Microphysics in astrophysical plasmas. Space Science Reviews, 2013 in press doi: TBD

J. D. Scudder, T. L. Aggson, A. Mangeney, C. Lacombe, and C. C. Harvey. The resolved layer of a collisionless, high beta, supercritical, quasi-perpendicular shock wave. I - RankineHugoniot geometry, currents, and stationarity. Journal of Geophysical Research, 91:1101911052, October 1986a. doi: 10.1029/JA091iA10p11019.

J. D. Scudder, A. Mangeney, C. Lacombe, C. C. Harvey, and C. S. Wu. The resolved layer of a collisionless, high beta, supercritical, quasi-perpendicular shock wave. III - Vlasov electrodynamics. Journal of Geophysical Research, 91:11075-11097, October 1986b. doi: 10.1029/JA091iA10p11075.

M. J. Seaton. Excitation of coronal lines by proton impact. Monthly Notices of the Royal Astronomical Society, 127:191-194, December 1964. doi: .

J. M. Shull and C. F. McKee. Theoretical models of interstellar shocks. I - Radiative transfer and UV precursors . Astrophysical Journal, 327:191-149, January 1979. doi: 10.1086/ 156712.

L. Sironi and A. Spitkovsky. Particle Acceleration in Relativsitic Magnetized Collisionless Electron-Ion Shocks. Astrophysical Journal , 726:75, January 2011. doi: 10.1088/ 0004-637X/726/2/75.

J. Skilling. Cosmic Ray Streaming. Nature, 258:687-688, December 1975. doi: 10.1038/ $258687 \mathrm{a} 0$.

P. Slane, B. M. Gaensler, T. M. Dame, J. P. Hughes, P. Plucinsky, and A. Green. Nonthermal X-Ray Emission from the Shell-Type Supernova Remnant G347.3-0.5. Astrophysical Journal, 525:357-367, November 1999. doi: 10.1086/307893.

R. C. Smith, R. P. Kirshner, W. P. Blair, and P. F. Winkler. Six Balmer Dominated Supernova Remnants. Astrophysical Journal, 375:652-662, July 1991. doi: 10.1086/170228.

R. C. Smith, J. C. Raymond, and J. M. Laming. High-resolution spectroscopy of Balmerdominated shocks in the Large Magellanic Cloud. Astrophysical Journal, 4220:286-293, January 1994. doi: 10.1086/1735581.

J. Sollerman, P. Ghavamian, P. Lundqvist, and R. C. Smith. High resolution spectroscopy of Balmer-dominated shocks in the RCW 86, Kepler and SN 1006 supernova remnants. Astronomy and Astrophysics, 407:249-257, August 2003. doi: 10.1051/0004-6361:20030839.

L. Spitzer. Physics of Fully Ionized Gases. New York: Interscience, 1964. doi: .

T. Tanaka, Y. Uchiyama, F. A. Aharonian, T. Takahashi, A. Bamba, J. S. Hiraka, J. Kataoka, T. Kishishita, M. Kokubun, K. Mori, K. Nakazawa, R. Petre, H. Tajima, and S. Watanabe. Study of Nonthermal Emission from SNR RX J1713.7-3946 with Suzaku. Astrophysical Journal, 685:988-1004, October 2008. doi: 10.1086/591020.

R. A. Treumann. Fundamentals of collisionless shocks for astrophysical application. I. Nonrelativsitic shocks Astronomy and Astrophysics Review, 17:409-535, December 2009 doi: 10.1007/s00159-009-0024-2.

D. Tseliakhovich, C. M. Hirata, and K. Heng. Excitation and charge transfer in $\mathrm{H}_{-} \mathrm{H}^{+}$collisions at 5-80 keV and application to astrophysical shocks. Monthly Notices of the Royal Astronomical Society, 422:2357-2371, May 2012. doi: 10.1111/j.1365-2966.2012.20787.x.

T. Umeda, Y. Kidani, S. Matsukiyo, and R. Yamazaki. Modified two-stream instability at perpendicular collisionless shocks: Full particle simulations. Journal of Geophysical Research, 117:A03206, March 2012. doi: 10.1029/2011JA017182.

T. Umeda, Y. Kidani, S. Matsukiyo, and R. Yamazaki. Microinstabilities at perpendicular collisionless shocks: A comparison of full particle simulations with different ion to electron mass ratio. Physics of Plasmas, 19:042109, April 2012. doi: 10.1063/1.3703319.

M. van Adelsberg, K. Heng, R. McCray, and J. C. Raymond. Spatial Structure and Collisionless Electron Heating in Balmer-dominated Shocks. Astrophysical Journal, 689:1089-1104, December 2008. doi: 10.1086/592680.

J. Vink and J. M. Laming. On the Magnetic Fields and Particle Acceleration in Cassiopeia A. Astrophysical Journal, 584:758-769, February 2003. doi: 10.1086/345832.

J. Vink, R. Yamazaki, E. A. Helder, and K. M. Schure. The Relation Between Post-shock Temperature, Cosmic-ray Pressure, and Cosmic-ray Escape for Non-relativistic Shocks. Astrophysical Journal, 722:1727-1734, October 2010. doi: 10.1088/0004-637X/722/2/1727.

A. Y. Wagner, J.-J. Lee, J. C. Raymond, T. W. Hartquist, and S. A. E. G. Falle. A CosmicRay Precursor Model for a Balmer-Dominated Shock in Tycho's Supernova Remnant. Astrophysical Journal, 690:1412-1423, January 2009. doi: 10.1088/0004-637X/690/2/1412. 
J. Warren, J. P. Hughes. Raising the Dead: Clues to Type Ia Supernova Physics from the Remnant 0509-67.5. Astrophysical Journal, 608:261-273, June 2004. doi: 10.1086/392528.

J. S. Warren, J. P. Hughes, C. Badenes, P. Ghavamian, C. F. McKee, D. Moffett, P. Plucinsky, C. E. Rakowski, E. Reynoso, and P. Slane. Cosmic-Ray Acceleration at the Forward Shock in Tycho's Supernova Remnant: Evidence from Chandra X-Ray Observations. Astrophysical Journal, 634:376-389, November 2005. doi: 10.1086/496941.

B. J. Williams, W. P. Blair, J. M. Blondin, K. J. Borkowski, P. Ghavamian, K. S. Long, J. C. Raymond, S. P. Reynolds, J. Rho, and P. F. Winkler. RCW 86: A Type Ia Supernova in a Wind-Blown Bubble. Astrophysical Journal, 741:96, November 2011. doi: 10.1088/ 0004-637X/741/2/96.

C. S. Wu, D. Winske, M. Tanaka, K. Papadopoulos, K. Akimoto, C. C. Goodrich, Y. M. Zhou, S. T. Tsai, P. Rodriguez, and C. S. Lin. Microinstabilities associated with a high Mach number, perpendicular bow shock. Space Science Reviews, 37:63-109, January 1984. doi: 10.1007/BF00213958.

S. A. Zhekov, R. McCray, D. Dewey, C. R. Canizares, K. J. Borkowski, D. N. Burrows, and S. Park High-Resolution X-Ray Spectroscopy of SNR 1987A: Chandra Letg and HETG Observations in 2007. Astrophysical Journal, 692:1190-1204, February 2009. doi: 10.1088/0004-637X/692/2/1190. 